\title{
A Novel Dynamic Model for Health Economic Analysis of Influenza Vaccination in the Elderly
}

\author{
Mark Mullikin · Litjen Tan · Jeroen P. Jansen • \\ Marc Van Ranst · Norbert Farkas · Eckhardt Petri
}

To view enhanced content go to www.infectiousdiseases-open.com

Received: April 10, 2015 / Published online: September 9, 2015

(C) The Author(s) 2015. This article is published with open access at Springerlink.com

\section{ABSTRACT}

Introduction: New vaccines are being developed to improve the efficacy of seasonal influenza immunization in elderly persons aged $\geq 65$ years. These products require clinical and economic evaluation to aid policy decisions.

Methods: To address this need, a two-part model has been developed, which we have applied to examine the potential clinical and

Electronic supplementary material The online version of this article (doi:10.1007/s40121-015-0076-8) contains supplementary material, which is available to authorized users.

M. Mullikin

Novartis Vaccines, Cambridge, MA, USA

L. Tan

Immunization Action Coalition, Saint Paul, MN, USA

J. P. Jansen

Redwood Outcomes, San Francisco, CA, USA

M. Van Ranst

KU Leuven, Rega Institute, Louvain, Belgium

N. Farkas

Novartis Vaccines, Basel, Switzerland

E. Petri $(\square)$

Novartis Vaccines, Marburg, Germany

e-mail: eckhardt.petri@novartis.com economic impact of vaccinating elderly persons with adjuvanted trivalent inactivated influenza vaccine (aTIV) relative to conventional trivalent (TIV) and quadrivalent (QIV) vaccines. We compared outcomes in the US population for (1) aTIV in persons aged $\geq 65$ years and QIV in all other age cohorts; (2) QIV in all cohorts; (3) TIV in all cohorts. Low, average, and high intensity seasons with low, average, and high vaccine match scenarios were compared. Probabilistic sensitivity analysis was conducted within each discrete scenario to explore the impact of variation in model inputs on potential outcomes.

Results: Assuming current vaccination coverage rates in the US population with (a) $25 \%$ better efficacy of adjuvanted versus non-adjuvanted vaccine against any strain and (b) $35 \%$ better efficacy of non-adjuvanted vaccine against matched $B$ versus mismatched B strains, use of aTIV in persons aged $\geq 65$ years and QIV in persons <65 years could reduce influenza cases by 11,166-1,329,200, hospitalizations by $1365-43,674$, and deaths by 421-11,320 versus use of QIV in all cohorts. These outcomes are reflected in a corresponding increase in quality-adjusted life-years (QALYs) 
of 3003-94,084. If the prevalence of mismatched influenza B was $>54.5 \%$ of all circulating strains, use of QIV in all cohorts would offset the clinical benefits of aTIV. Elderly aTIV or QIV vaccination was associated with improved outcomes over non-adjuvanted TIV in many of the scenarios, particularly in low match seasons of any intensity. Total cost savings (including direct and indirect healthcare costs plus productivity impacts) with aTIV in the elderly versus QIV in the whole population ranged from $\$ 27$ million (low intensity, low match) to $\$ 934$ million (high intensity, high match). Univariate sensitivity analysis of relative vaccine prices in the average intensity, average match scenario indicated that aTIV could be marginally cost saving relative to QIV at the currently published Medicare price for influenza vaccines offering enhanced efficacy in the elderly. Elderly vaccination with aTIV was associated with a higher overall cost compared with TIV in only two scenarios (low intensity with average or high match); the incremental cost/QALY relative to TIV was $\$ 9980$ in the average match scenario and $\$ 28,800$ in the high match scenario.

Conclusions: Vaccination of persons aged $\geq 65$ years with aTIV has the potential to provide clinical and economic benefit relative to QIV and TIV. The new model allows the assessment of various alternative strategies for available influenza vaccines.

Funding: Novartis Vaccines.

Keywords: Influenza; Elderly; Vaccine; Trivalent; Quadrivalent; Adjuvant; Vaccination; Economic; Clinical; Outcome

\section{INTRODUCTION}

The clinical and economic burden of influenza is significant, particularly in vulnerable populations, such as children, persons with compromised immune function, and the elderly. Notably, the risk of influenza-related complications increases with age [1-3], influenza-associated hospitalizations are most common among elderly aged $\geq 65$ years (especially in those with underlying medical conditions) [4-8], and up to $90 \%$ of influenza-attributable mortality is seen in this cohort [9-11].

Significant direct healthcare costs are linked to influenza in persons aged $\geq 65$ years [6]. These costs are driven by substantial numbers of influenza-related hospitalizations, and are increasing as the overall population ages [9]. Moreover, influenza-related hospitalizations in the elderly are associated with significant disability and impairments in activities of daily living. These effects have societal implications as they can be long lasting, and catastrophic disability after influenza hospitalization is a significant problem in this age cohort $[12,13]$. Influenza vaccination in the elderly provides substantial benefits [14], and past cost-benefit and cost-effectiveness studies from a number of countries have indicated that this intervention in this age cohort is always cost-effective and is frequently cost saving [15]. Influenza vaccination policy in most developed countries, therefore, targets people aged $\geq 65$ years as part of the effort to reduce the mortality and disability burden in this population [16].

The efficacy of conventional inactivated influenza vaccine [trivalent (TIV) or quadrivalent (QIV)] decreases with advancing age because of reduced production of vaccine-specific antibodies [17]. Attempts to increase immunogenicity have been made with alternatives such as high-dose TIV $(60 \mu \mathrm{g}$ of hemagglutinin (HA)/strain) [18] and an intradermal vaccine [19]. Constant evolution 
of the influenza strains in circulation further challenges vaccine protection with approximately $35 \%$ reduction in efficacy against mismatched versus matched strains $[20,21]$. As a result, QIV may offer benefit in seasons where the B lineage selected for TIVs does not match the dominant circulating strain [22]. Notably, however, TIV adjuvanted with the squalene-containing oil-in-water emulsion MF59 (aTIV) increases seroprotection [23, 24] and has been associated with a $25 \%$ reduction in risk of hospitalization for influenza or pneumonia versus TIV in the elderly population aged $\geq 65$ years [25]. Moreover, in the elderly, aTIV has been reported to provide better cross-reactivity against mismatched strains than conventional vaccine [26].

As the economic burden of influenza is disproportionately concentrated in the elderly [27], improvements in vaccine immunogenicity and efficacy in this group have economic implications that warrant investigation. Economic modeling is an important and generally accepted method for estimating the economic effects of an intervention [28]. A two-part epidemiologic and economic model was, therefore, developed to assess the clinical and economic impact of vaccination with aTIV in persons aged $\geq 65$ years and QIV in those aged $<65$ years versus QIV or TIV in all age cohorts.

\section{METHODS}

\section{Scenarios of Interest}

This analysis compared clinical and economic outcomes over a 1-year period in the United States (US) population for three vaccination strategies: (1) aTIV in those $\geq 65$ years (elderly) and QIV in all other age cohorts; (2) QIV in all cohorts; (3) TIV in all cohorts. We compared outcomes in nine discrete scenarios for low, average, and high intensity (i.e., transmissibility) seasons, factoring in low, average, and high vaccine match against circulating seasonal strains, in a $3 \times 3$ matrix. The analysis in this article is based on previously conducted studies, and does not involve any new studies of human or animal subjects performed by any of the authors.

The average match scenarios (based on the arithmetic means of strain circulation in the US from 1999-2000 through 2013-2014 [29-33]) were $48.3 \%, 30.4 \%, 11.0 \%$, and $10.3 \%$ for A/H3N2, A/H1N1, B/Victoria, and B/Yamagata, respectively. The low and high match scenarios were based on the 2000/2001 season, during which there was a very high prevalence of influenza $\mathrm{B} /$ Yamagata $(1.7 \%, 51.9 \%, 0 \%$, and $46.4 \%$ for A/H3N2, A/H1N1, B/Victoria, and $\mathrm{B} /$ Yamagata, respectively).

The TIV and aTIV vaccines contain the dominant circulating B strain (B/Yamagata) in the high match scenarios, but not in the low match scenarios. We also investigated a breakeven scenario for vaccine match, modeled for a low intensity season in which the low attack rate minimizes the clinical impact of vaccine efficacy. We assumed (a) $25 \%$ better efficacy of adjuvanted versus non-adjuvanted vaccine against any strain and (b) $35 \%$ better efficacy of non-adjuvanted vaccine against matched $B$ versus mismatched B strains.

\section{The Two-Part Model}

The model is programmed in Microsoft Excel, and consists of a compartmental, dynamic "epidemiologic module" to estimate the number of influenza cases and a tree-structured "outcomes module" to estimate complications, hospitalizations, deaths, 
life-years, quality-adjusted life-years (QALYs), and costs associated with influenza cases over a 1-year period (Fig. 1). The model assumes no adverse events of influenza vaccination or treatment, and mutual exclusivity of complications. It also does not consider the impact of strain mutation within a season, and recovered/protected patients remain immune for the duration of the simulation.

\section{Epidemiologic Module}

The "epidemiologic module" simulates the transmission of seasonal influenza with a susceptible-infectious-recovered/protected/ removed (SIR) model consisting of 6 compartments (Fig. 2). It estimates the dynamic changes over one year with or without vaccination (three unique age-stratified strategies can be defined) in the uninfected, infected with influenza, and the recovered/protected/removed populations. The population size is constant and proportions in the different compartments (or health states) can vary by age cohort i (0-3 years, 4-6, 7-9, $10-19,21-34,35-49,50-64,65-69,70+)$. No patients enter or leave the system over the modeled year, and proportions in each compartment sum to 1. Patients recovered/ protected/removed from infection (including patients who die as a result of infection) are fully immune and, therefore, cannot be re-infected or infect others for the remainder of the simulation (effectively removed from the simulation).

As illustrated in Fig. 2, in the proportion of the population that will be vaccinated $\left(C v_{i}\right)$, some people who receive vaccination are susceptible $\left(S_{i}\right)$ and some are already protected by previous vaccination or influenza exposure $\left(R_{i}\right)$. Among those not yet protected $\left(S_{i}\right)$, vaccination can be successful (the individual enters compartment $V R_{i}$ ) or unsuccessful (enters compartment $\left.V S_{i}\right)$. Vaccine efficacy $\left(1-P_{i}\right)$ determines distributions over the $V S_{i}$ and $V R_{i}$ compartments. All persons already protected before vaccination $\left(R_{i}\right)$ will move to compartment $V R_{i}$.

The rate at which individuals in age cohort $i$ transition from susceptible $S_{i}$ to infected/ infectious $I_{i}$ is the force of infection, $\lambda_{i}$. Patients who are not fully protected after

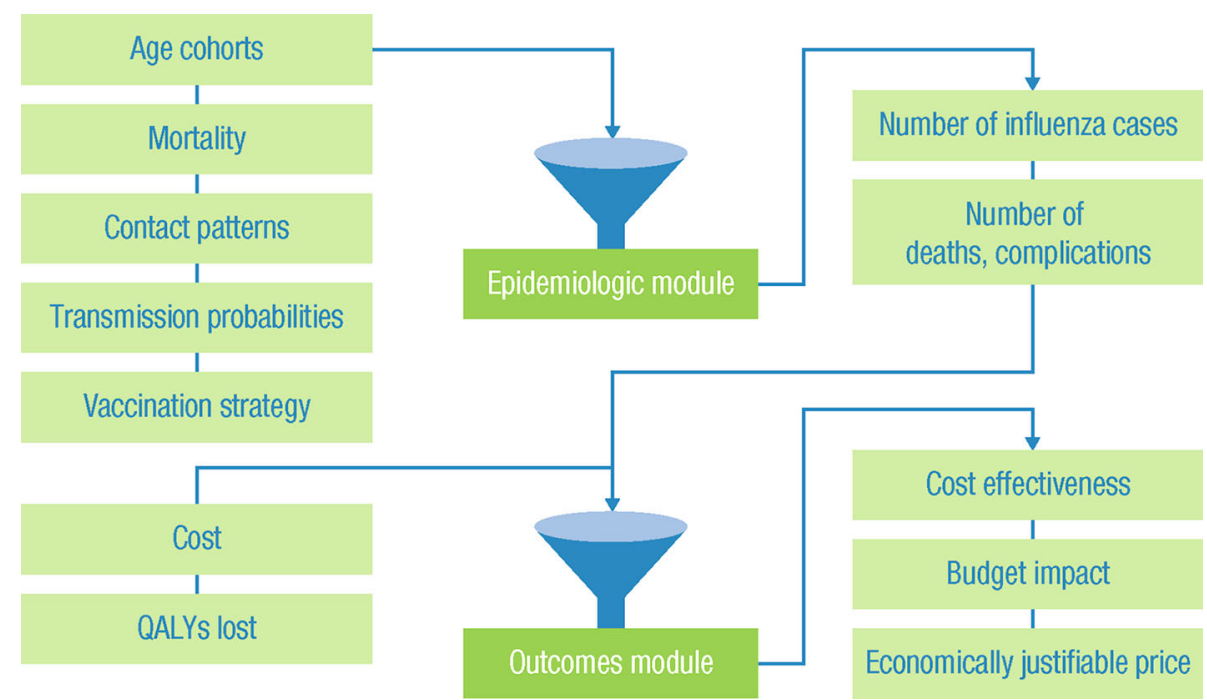

Fig. 1 The two-part epidemiologic and economic model. QALY quality-adjusted life-year 


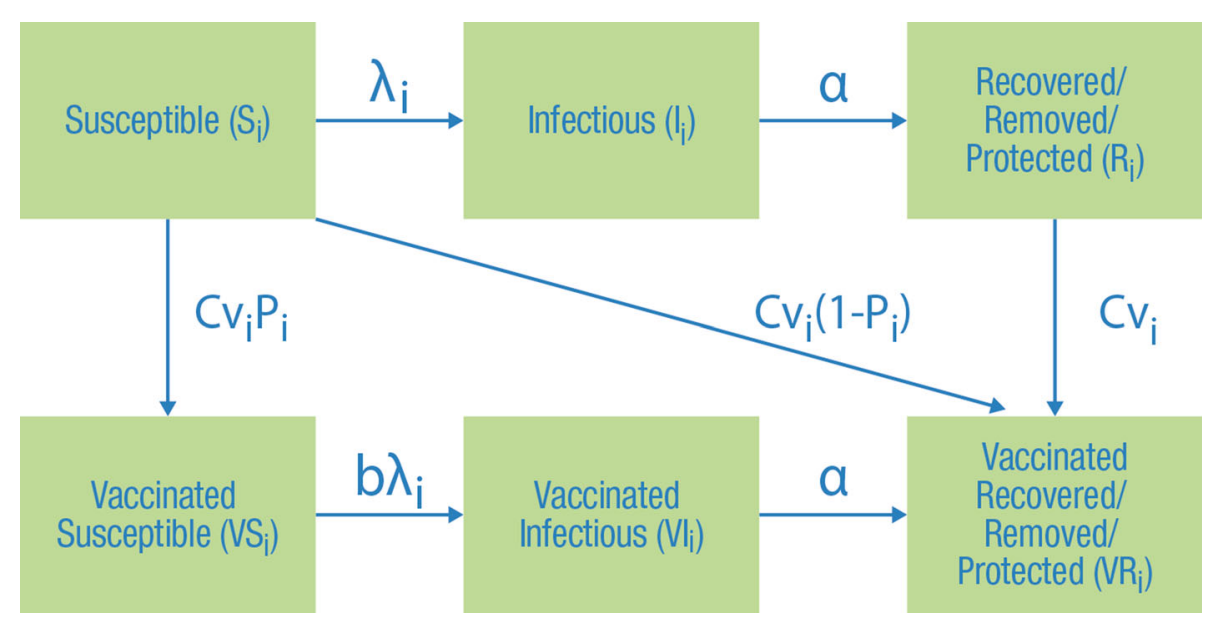

Fig. 2 The susceptible-infectious-recovered (SIR) compartment model for seasonal influenza. $S_{i}(t)=$ Fraction of susceptible individuals to influenza in age cohort $i$ at time $t . I_{i}(t)=$ Fraction of infectious individuals in age cohort $i$ at time $t . R_{i}(t)=$ Fraction of recovered/protected/ removed from influenza in age cohort $i$ at time t. $V S_{i}(t)=$ Fraction of vaccinated but still susceptible for influenza in age cohort $i$ at time $t . V I_{i}(t)=$ Fraction of

vaccination $\left(V S_{i}\right)$ can be infected, but at a rate lower than or equal to unvaccinated individuals, represented by $b \lambda_{i}$, where $0<b \leq 1$. Upon infection with influenza, individuals recover according to rate $\alpha$ and move to $R_{i}$ (or $V R_{i}$ ); they are immune to further influenza infection for the remainder of the year and, therefore, cannot infect others (i.e., effectively removed from the simulation).

Changes in the proportion of patients over the compartments are defined by the differential equations:

$$
\begin{aligned}
& \frac{\mathrm{d} S_{i}(t)}{\mathrm{d} t}=-S_{i}(t) \lambda_{i}(t)-S_{i}(t) C v_{i} P_{i} \\
&-S_{i}(t) C v_{i}\left(1-P_{i}\right) \\
& \frac{\mathrm{d} V S_{i}(t)}{\mathrm{d} t}=-V S_{i}(t) b \lambda_{i}(t)+S_{i}(t) C v_{i} P_{i} \\
& \frac{\mathrm{d} I_{i}(t)}{\mathrm{d} t}=S_{i}(t) \lambda_{i}(t)-I_{i}(t) \alpha
\end{aligned}
$$

influenza cases despite being vaccinated in age cohort $i$ at time $t . V R_{i}(t)=$ Fraction of vaccinated recovered/protected/removed in age cohort $i$ at time $t . C v_{i}=$ Vaccine coverage in age cohort i. $P_{i}=$ Probability of unsuccessful vaccination in age cohort $i . \lambda_{i}(t)=$ Force of infection in age cohort $i$ at time $t . b \lambda_{i}(t)=$ Force of infection in vaccinated cohort in age cohort $i$ at time $t ; \alpha=$ Recovery rate

$\frac{\mathrm{d} V I_{i}(t)}{\mathrm{d} t}=V S_{i}(t) b \lambda_{i}(t)-V I_{i}(t) \alpha$

$\frac{\mathrm{d} R_{i}(t)}{\mathrm{d} t}=I_{i}(t) \alpha-R_{i}(t) C v_{i}$

$\frac{\mathrm{d} V R_{i}(t)}{\mathrm{d} t}=V I_{i}(t) \alpha+S_{i}(t) C v_{i}\left(1-P_{i}\right)+R_{i}(t) C v_{i}$

The force of infection $\lambda_{i}(t)$ is the rate at which susceptible individuals in age cohort $i$ are infected at time $t$. It is the summation of the rates of infection at time $t$ from all infected/ infectious in age cohorts $j$ :

$\lambda_{i}(t)=\sum_{j} \lambda_{i j}(t)$

where:

$\lambda_{i j}(t)=\sigma \cdot \gamma_{i j} \cdot \varphi_{i} \cdot \xi_{j} \cdot I_{j}(t)=\beta_{i j} \cdot I_{j}(t)$

where:

$\sigma$ is the transmissibility parameter. 
$\gamma_{i j}$ is the average number of contacts per day between susceptible individuals in age cohort $i$ with infectious individuals in age cohort $j$.

$\varphi_{i}$ is the susceptibility of a susceptible individual in age cohort $i$.

$\zeta_{j}$ is the infectivity of an infective individual in age cohort $j$.

$I_{j}(t)$ is the fraction of infectious individuals in age cohort $j$ at time $t$.

$\beta_{i j}$ is the rate of infection of susceptible individuals in age cohort $i$ by infectious individuals in cohort $j$ (referred to as the WAIFW or Who Acquired Infection from Whom matrix; see Electronic Supplementary Material (ESM) for further discussion of derivation and implications for transmission dynamics).

Inputs of the model include age-stratified population [34], an age-stratified contact matrix $(\gamma)$ representing interactions between individuals in the population leading to spread of the disease ([35], adapted to fit this model's age structure; Table S1), duration of infection $(1 / v)$ (assumed gamma distribution with mean of 4 days and standard deviation of 1 day [36-38], strain circulation [29-33], vaccine coverage by age [39], and vaccination efficacy by strategy by level of vaccine match to circulating strains by age [20, 2125,40$]$. The susceptibility $\varphi_{i}$, infectivity $\zeta_{j}$, and transmission $\sigma$ parameters are derived through a calibration process described below and in the ESM. Full details of these inputs are shown in Tables S1-S8.

\section{Outcomes Module}

The "outcomes module" calculates the outcomes associated with influenza according to a tree structure (Fig. 3; Tables 1, 2, 3 [27, 39, 41-48]). Upon infection with influenza, a person can experience symptoms (first chance node and corresponding split path). If symptoms are present, a patient might seek medical consultation (second chance node). Given medical consultation, antivirals and/or other drugs might be prescribed (third and fourth chance nodes). The conditional probabilities of influenza-related complications can be influenced by the prescription of antivirals and other drugs. We assumed the risk of complications to be the same with and without vaccination (i.e., the risk of complications is entirely dependent on whether an individual is infected with influenza). The probability of hospitalization or death in the presence of a complication is the result of the calibration process described below. The conditional probabilities with their sources are shown in Table S9.

Direct costs (US\$) for medical care, including medical consultation, prescriptions for antivirals and other drugs [42], and costs of complications with and without hospitalization [42, 43, 49], were included (Table 1). Indirect costs covered productivity losses based on the severity of complications [27]. QALY reductions for the duration of symptoms of influenza and its complications (Table 2) and QALY losses due to life-years lost due to fatal complications were also included (Table 3). Life-years and QALYs lost were discounted at 3\% per annum.

Expected outcomes and costs for each new influenza case were calculated by multiplying the probability of each event as reflected in the final branches of the tree with the corresponding outcomes and costs. In combination with the incidence of influenza over the course of a 1-year period as estimated with the "epidemiologic module", the expected outcomes associated with influenza over this period were obtained. 


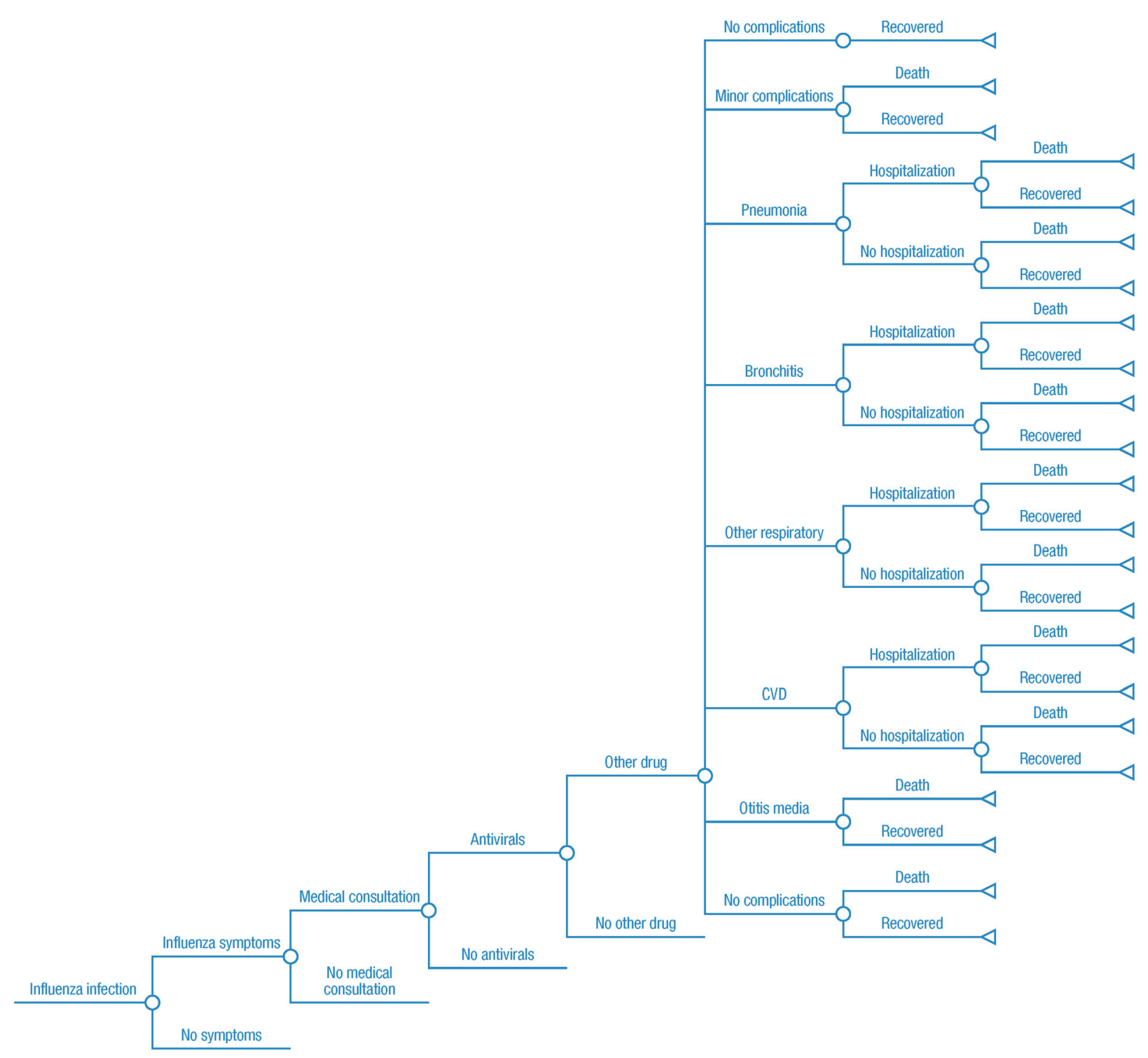

Fig. 3 Outcomes associated with influenza. CVD cardiovascular disease

\section{Calibration of the Model}

The model was calibrated to the age-stratified number of cases based on age-stratified estimated gross attack rates [27] and estimates of influenza incidence $[27,29,50]$ by adjusting a factor for transmissibility $(\sigma)$ and age-specific factors for susceptibility $\left(\varphi_{i}\right)$. Conditional probabilities for hospitalization and death by complication according to age were then calibrated to literature-based estimates [27] (see the ESM for further detail).

\section{Estimation of Model Outcomes}

For each scenario and vaccination strategy of interest, we conducted a probabilistic analysis (second-order Monte Carlo simulation) by 
Table 1 Input data: costs used in the model

\begin{tabular}{|c|c|c|}
\hline Variable & Estimate (\$US) & Source \\
\hline \multicolumn{3}{|c|}{ Vaccine acquisition } \\
\hline TIV & 9.45 & CDC [39] \\
\hline aTIV & 13.65 & Assumption \\
\hline QIV & 13.65 & Assumption \\
\hline \multicolumn{3}{|c|}{ Vaccine administration } \\
\hline All ages & 21.00 & Prosser $[41]$ \\
\hline \multicolumn{3}{|c|}{ Antiviral cost } \\
\hline All ages & 92.35 & Talbird et al. [42] \\
\hline \multicolumn{3}{|c|}{ Medical consultation } \\
\hline All ages & 98.79 & Talbird et al. [42] \\
\hline \multicolumn{3}{|c|}{ Complications } \\
\hline \multicolumn{3}{|c|}{ Pneumonia without hospitalization } \\
\hline All ages & 206 & Talbird et al. [42] \\
\hline \multicolumn{3}{|c|}{ Pneumonia with hospitalization } \\
\hline $0-19$ & 5513 & Talbird et al. [42] \\
\hline $20-64$ & 14,828 & \\
\hline$\geq 65$ & 14,137 & \\
\hline \multicolumn{3}{|c|}{ Bronchitis without hospitalization } \\
\hline All ages & 221 & Talbird et al. [42] \\
\hline \multicolumn{3}{|c|}{ Bronchitis with hospitalization } \\
\hline $0-19$ & 3906 & Talbird et al. [42] \\
\hline $20-64$ & 7449 & \\
\hline$\geq 65$ & 8834 & \\
\hline \multicolumn{3}{|c|}{ Other respiratory without hospitalization } \\
\hline All ages & 221 & Talbird et al. [42] \\
\hline \multicolumn{3}{|c|}{ Other respiratory with hospitalization } \\
\hline $0-19$ & 3906 & Talbird et al. [42] \\
\hline $20-64$ & 7449 & \\
\hline$\geq 65$ & 8834 & \\
\hline \multicolumn{3}{|c|}{ CVD without hospitalization } \\
\hline All ages & 2711 & American Heart Association [43] \\
\hline \multicolumn{3}{|c|}{ CVD with hospitalization } \\
\hline All ages & 6017 & American Heart Association [43] \\
\hline
\end{tabular}


Table 1 continued

\begin{tabular}{lll}
\hline Variable & Estimate (\$US) Source
\end{tabular}

Otitis media

$$
\text { All ages }
$$

Lost productivity costs

Without complications

$\begin{array}{ll}0-3 & 145 \\ 4-6 & 97 \\ 7-19 & 73 \\ 20-64 & 68 \\ 65+ & 145\end{array}$

Extra loss due to minor complications

$\begin{array}{ll}0-3 & 183 \\ 4-6 & 209 \\ 7-9 & 191 \\ 10-19 & 186 \\ 20-49 & 167 \\ 50-64 & 386 \\ 65+ & 832\end{array}$

Extra loss due to serious complications (pneumonia, bronchitis, CVD)

$\begin{array}{ll}0-3 & 1333 \\ 4-6 & 1576 \\ 7-9 & 1520 \\ 10-19 & 1603 \\ 20-49 & 1934 \\ 50-64 & 2411 \\ 65+ & 2256\end{array}$

Otitis media
All ages
0
Assumed no additional days lost

Molinari et al. [27]; weighted average of non-high-risk and high-risk outpatient visit

$\overline{a T I V}$ adjuvanted trivalent inactivated influenza vaccine, $C D C$ Centers For Disease Control And Prevention, $C V D$ cardiovascular disease, $Q I V$ Quadrivalent inactivated influenza vaccine, $T I V$ trivalent inactivated influenza vaccine

which variation in the model input parameters (summarized with probability density functions) was propagated through the model to obtain distributions for the following outcomes of interest: number of influenza cases, complications, deaths, life-years lost, 
Table 2 Input data: QALY loss with influenza and impact of events

\begin{tabular}{llllll}
\hline Variable & Estimate & Low & High & Distribution & Source/comments \\
\hline Minor complications & 0.00000 & 0.00000 & 0.00000 & Gamma & Assumption \\
Pneumonia & 0.01041 & 0.00674 & 0.01487 & Gamma & Lee et al. [44] \\
Bronchitis & 0.00904 & 0.00585 & 0.01291 & Gamma & Lee et al. [44] \\
Other respiratory & 0.00904 & 0.00585 & 0.01291 & Gamma & Lee et al. [44] \\
CVD & 0.10000 & 0.06471 & 0.14284 & Gamma & Dyer et al. [45] \\
Otitis media & 0.01382 & 0.00894 & 0.01974 & Gamma & Prosser et al. [46] \\
\hline
\end{tabular}

$C V D$ cardiovascular disease, $Q A L Y$ quality-adjusted life-year

Table 3 Input data: life expectancy and QALY loss due to death

\begin{tabular}{lllllll}
\hline Age & $\begin{array}{l}\text { Discounted life-years lost } \\
\text { when dying } \\
\text { Estimate }\end{array}$ & \multicolumn{3}{l}{ Discounted QALYs lost when dying } & Source/comments \\
\cline { 2 - 6 } & Estimate & Low & High & Distribution & \\
\hline $0-3$ & 30.8 & 27.7 & 25.0 & 30.5 & Normal & EQ-5D assumed 0.9 over rest of life \\
$4-6$ & 30.5 & 27.5 & 24.8 & 30.2 & Normal & EQ-5D assumed 0.9 over rest of life \\
$7-9$ & 30.2 & 27.2 & 24.5 & 29.8 & Normal & EQ-5D assumed 0.9 over rest of life \\
$10-19$ & 29.3 & 26.4 & 23.8 & 29.0 & Normal & EQ-5D assumed 0.9 over rest of life \\
$20-34$ & 27.2 & 24.5 & 22.1 & 26.9 & Normal & EQ-5D assumed 0.9 over rest of life \\
$35-49$ & 23.5 & 21.1 & 19.1 & 23.2 & Normal & EQ-5D assumed 0.9 over rest of life \\
$50-64$ & 18.3 & 15.8 & 14.2 & 17.3 & Normal & Fryback et al. [47] adjusted average for \\
& & 12.0 & 10.9 & 13.2 & Normal & Fryback et al. [47] adjusted average for \\
$65-69$ & 14.2 & & & & & $\begin{array}{c}\text { remaining life-years } \\
70+\end{array}$ \\
& 7.1 & 5.9 & 5.4 & 6.5 & Normal & $\begin{array}{c}\text { Fryback et al. [47] adjusted average for } \\
\text { remaining life-years }\end{array}$ \\
\hline
\end{tabular}

Source: life expectancy: [48]

$C D C$ Centers for Disease Control and Prevention, QALY Quality-adjusted life-year

QALYs lost, and costs. The distributions for these outcomes were summarized with the mean ("probabilistic mean"), low (2.5th percentile), and high (97.5th percentile) estimates.

The model output was further used to estimate budget impacts and cost-effectiveness of the competing interventions.

\section{RESULTS}

The model outputs for the low, average, and high intensity modeled seasons with low, average, and high vaccine match are summarized in Tables 4, 5, 6 and Figs. 4 and 5. Assuming (a) $25 \%$ better efficacy of adjuvanted versus non-adjuvanted vaccine against any 
strain and (b) $35 \%$ better efficacy of non-adjuvanted vaccine against matched $\mathrm{B}$ versus mismatched B strains, clinical benefits of aTIV vaccination in the population $\geq 65$ years and QIV in the population $<65$ years suggested by the model (versus vaccination of the whole population with QIV) included reductions in influenza cases of 11,166-1,329,200 (low estimate reflects probabilistic mean in low intensity, low match scenario; high estimate reflects probabilistic mean in high intensity, high match scenario), 1365-43,674 fewer hospitalizations, and 421-11,320 fewer deaths (Table 4). These clinical outcomes were reflected in gains of 3003-94,084 QALYs. As illustrated in Fig. 4, elderly vaccination with aTIV or QIV was associated with improved outcomes over non-adjuvanted TIV in many of the scenarios, particularly in low match seasons of any intensity. Moreover, in all scenarios, elderly vaccination with aTIV was shown by the model to be at least as effective as QIV in reducing cases and other clinical outcome rates (Fig. 4; Tables 4, 5). The number of influenza cases (95\% Credible Interval $[\mathrm{CrI}]$ ) when vaccinating all age cohorts with TIV ranged from 5.6 million (0.0-35.7) in the low intensity, high match scenario to 45.9 million (1.4-71.4) in the high intensity, low match scenario. Total costs (95\% CrI) when vaccinating all age cohorts with TIV ranged from $\$ 6.2$ billion (3.8-16.4) in the low intensity, high match scenario to $\$ 19.8$ billion (4.9-29.4) in the high intensity, low match scenario.

The breakeven analysis, which modeled a low intensity season in which the difference between elderly aTIV and QIV in number of cases was as close to zero as possible, showed that the prevalence of circulating mismatched strains would have to exceed $54.5 \%$ for QIV to offset the benefits of aTIV.
The potential impact of aTIV versus QIV vaccination in the elderly on costs was split evenly between direct savings ranging from $\$ 15$ million (low intensity, low match) to $\$ 475$ million (high intensity, high match) and indirect savings from $\$ 12$ million to \$459 million (Fig. 5; Table 6). Elderly aTIV vaccination was associated with increased overall cost over TIV in only two scenarios (low intensity with average or high match) but still represented good value for money with an incremental cost/QALY relative to TIV of $\$ 9980$ in the average match scenario and $\$ 28,800$ in the high match scenario. In both these scenarios, however, elderly aTIV dominated QIV with lower cost $(-\$ 171,071 ;-\$ 222,553)$ and higher QALYs $(14,676 ; 17,136)$. Univariate sensitivity analysis of relative vaccine prices in the average intensity, average match scenario indicated that aTIV could be marginally cost saving relative to QIV at current Medicare prices for influenza vaccines (Fig. 6 [51-53]).

Inspection of variability of results across scenarios showed that the outputs were most sensitive to vaccine match, followed by season intensity. The probabilistic nature of the model was illustrated by the probabilistic mean, low, and high estimates for each outcome within each discrete scenario, reflecting variation of inputs in observational studies and surveillance data.

\section{DISCUSSION}

We demonstrate that vaccination of persons aged $\geq 65$ years with aTIV has the potential to provide clinical and economic benefit relative to QIV and TIV. The dynamic model described herein can be used to inform policy decisions regarding seasonal influenza vaccination. Similar dynamic compartmental models have 


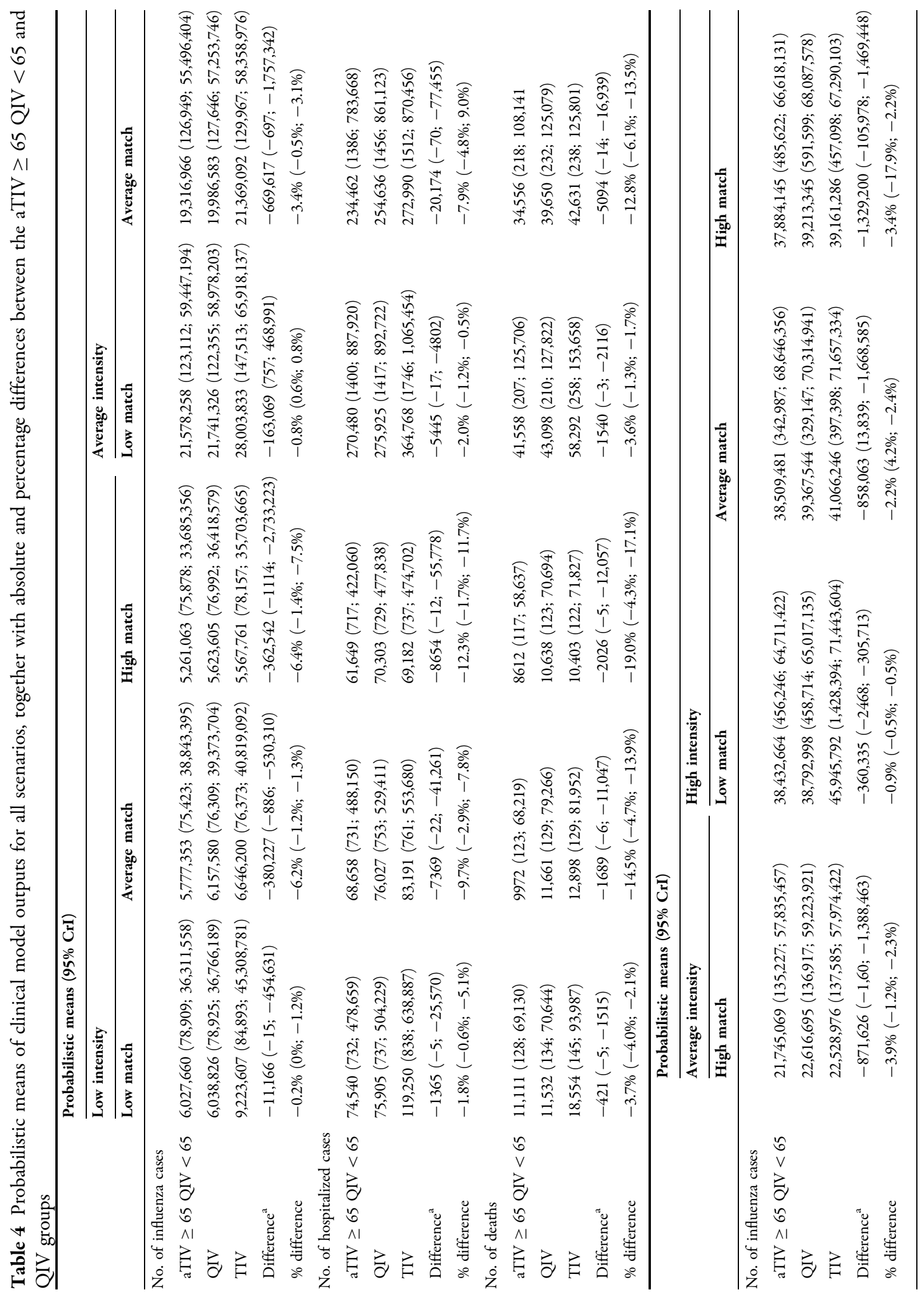




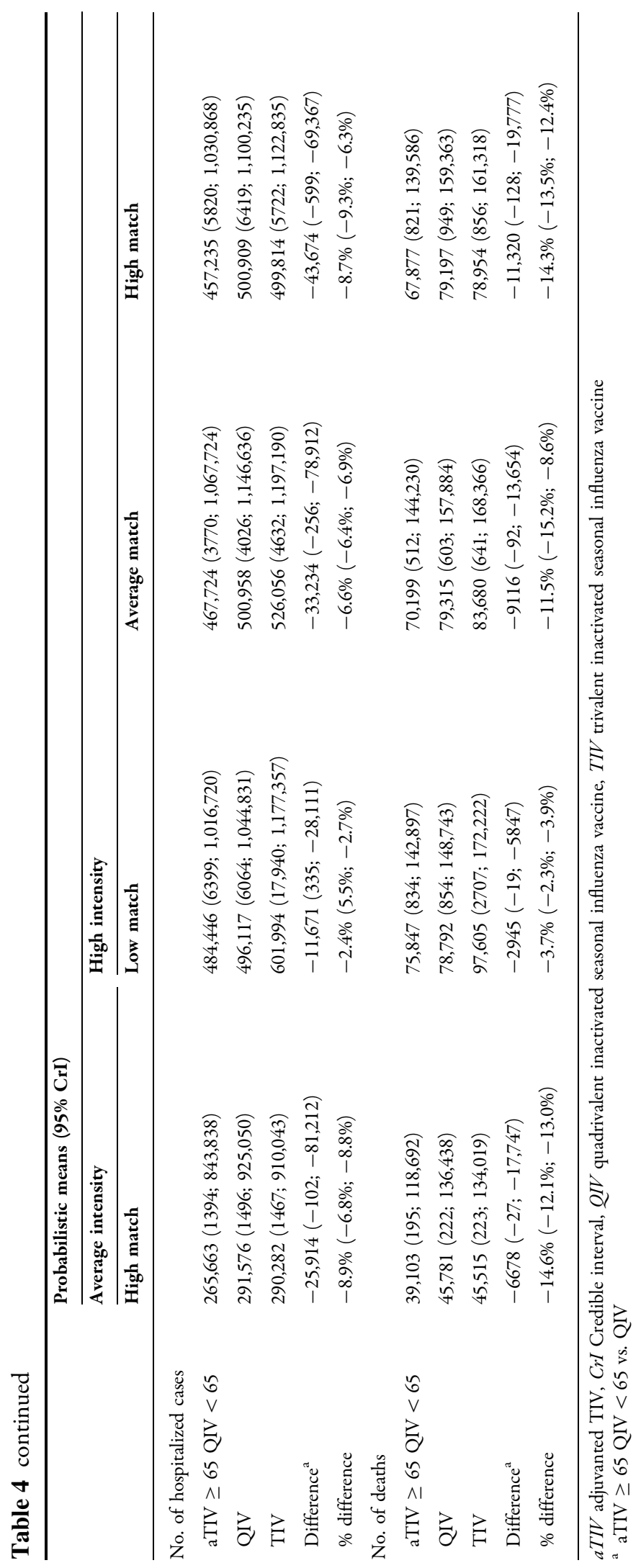




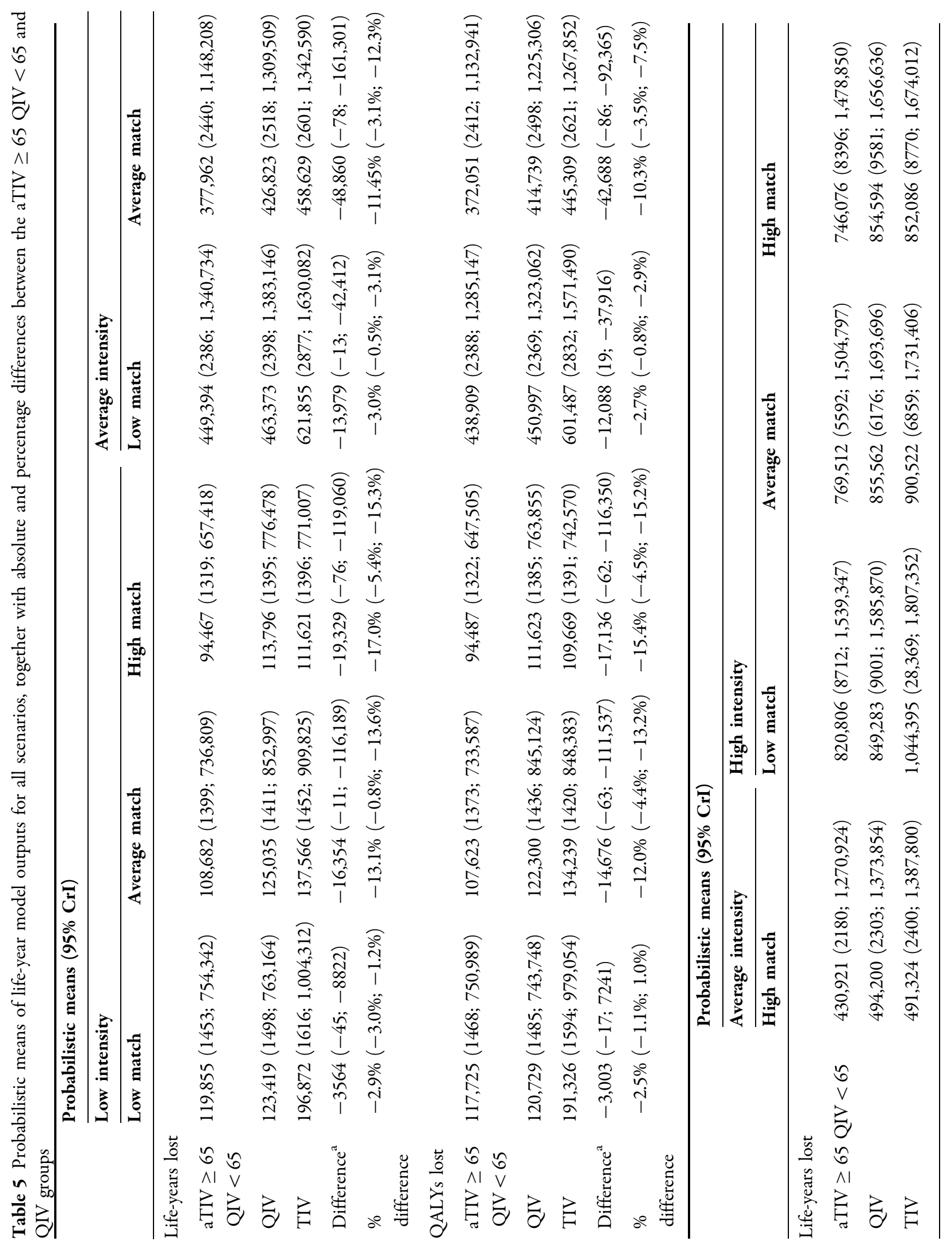




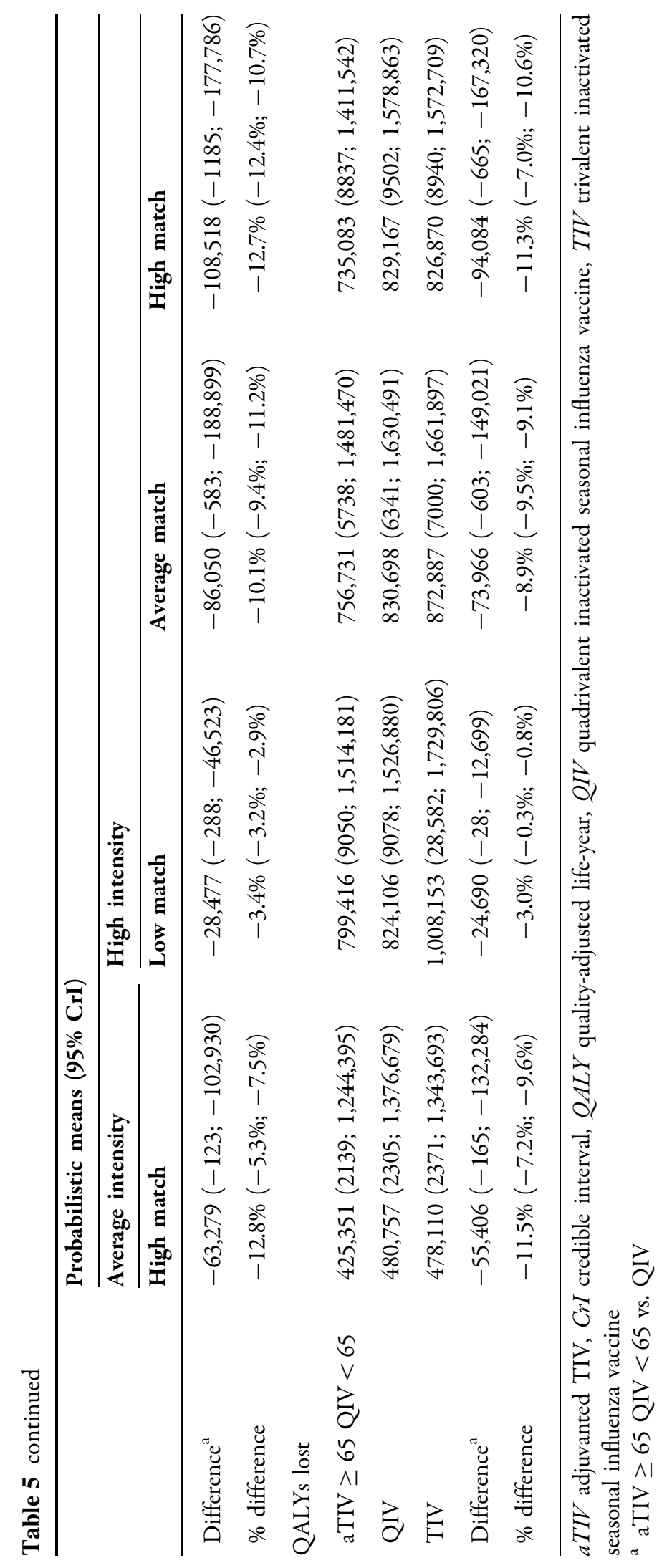




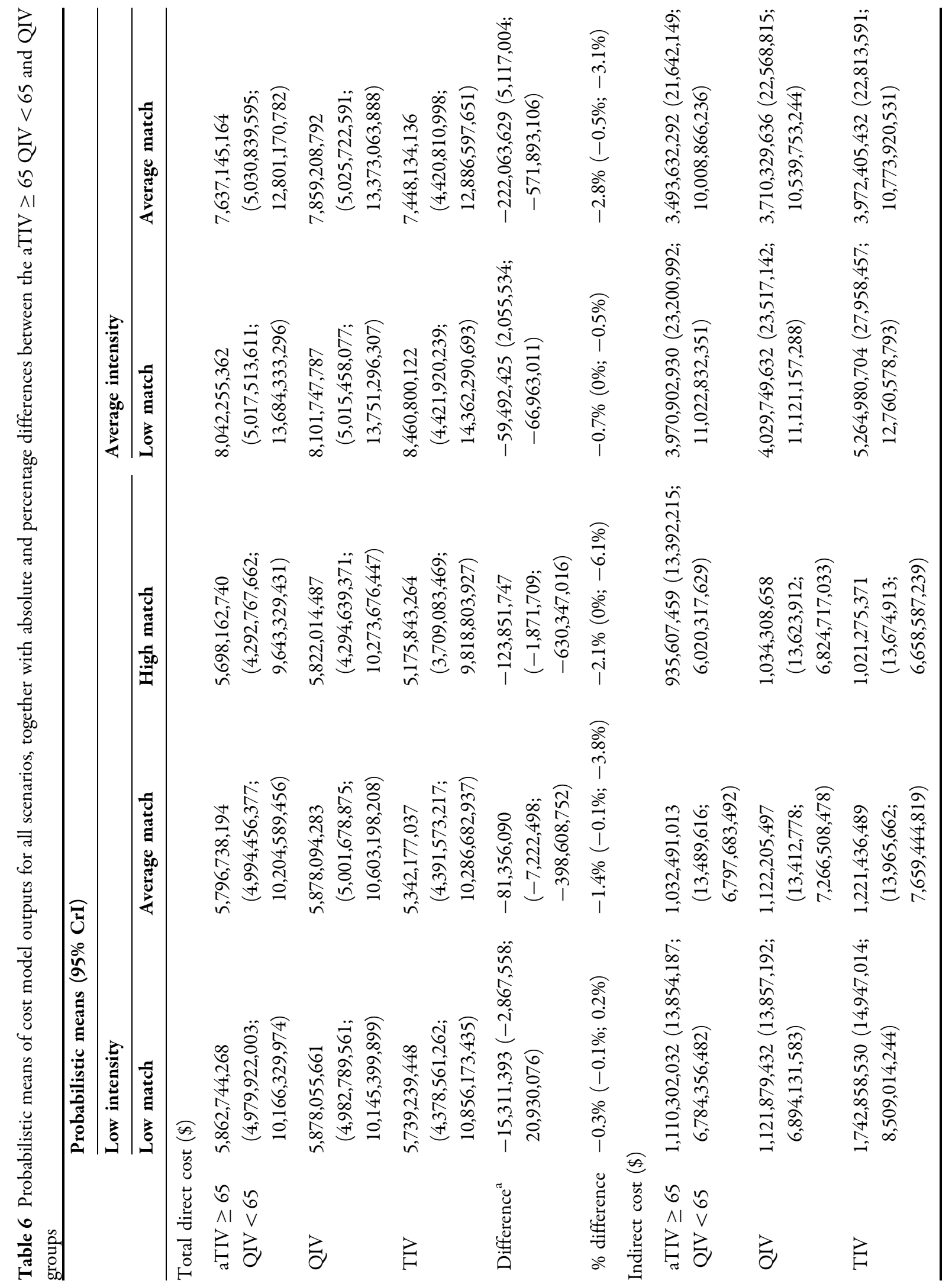




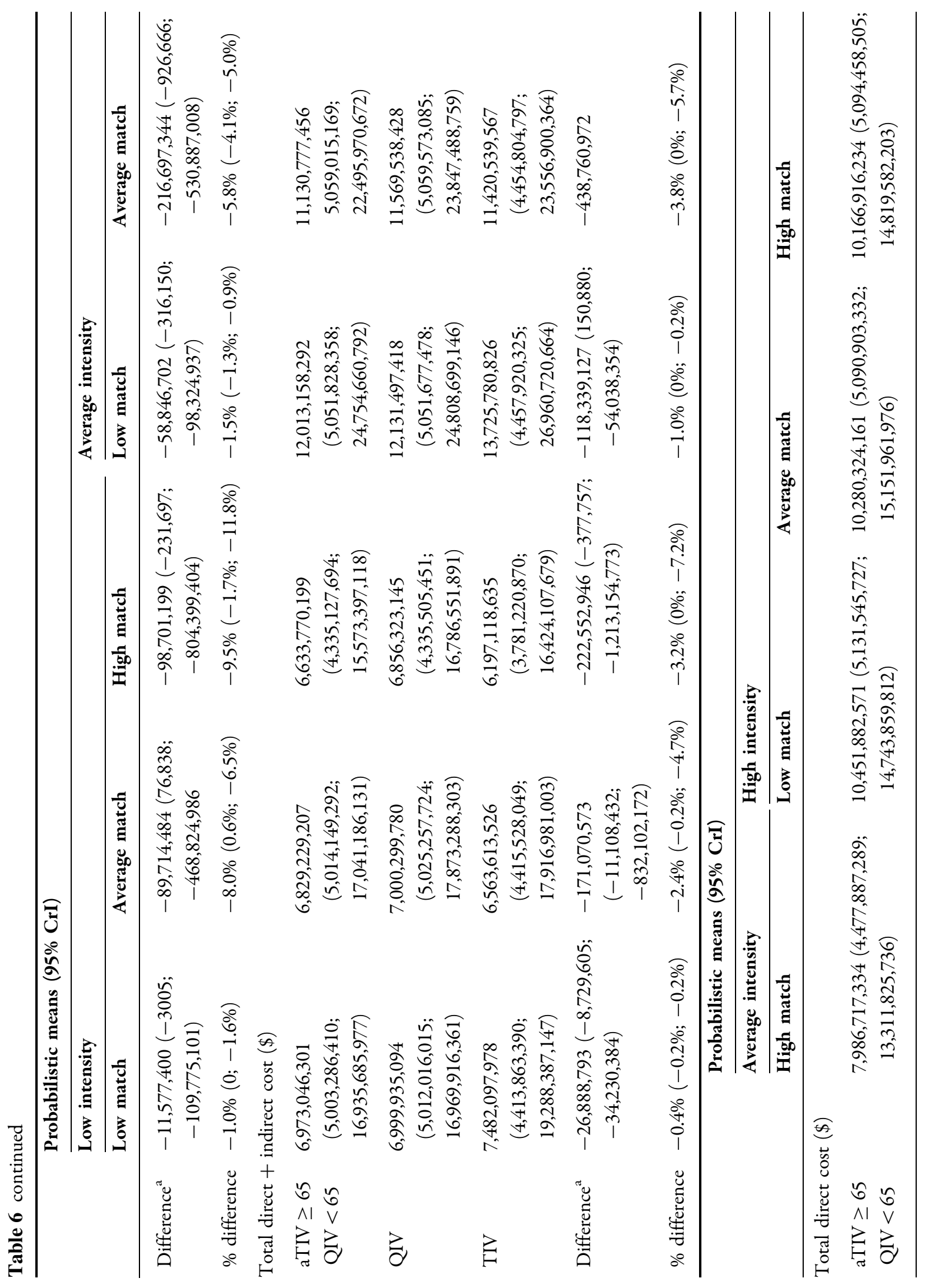




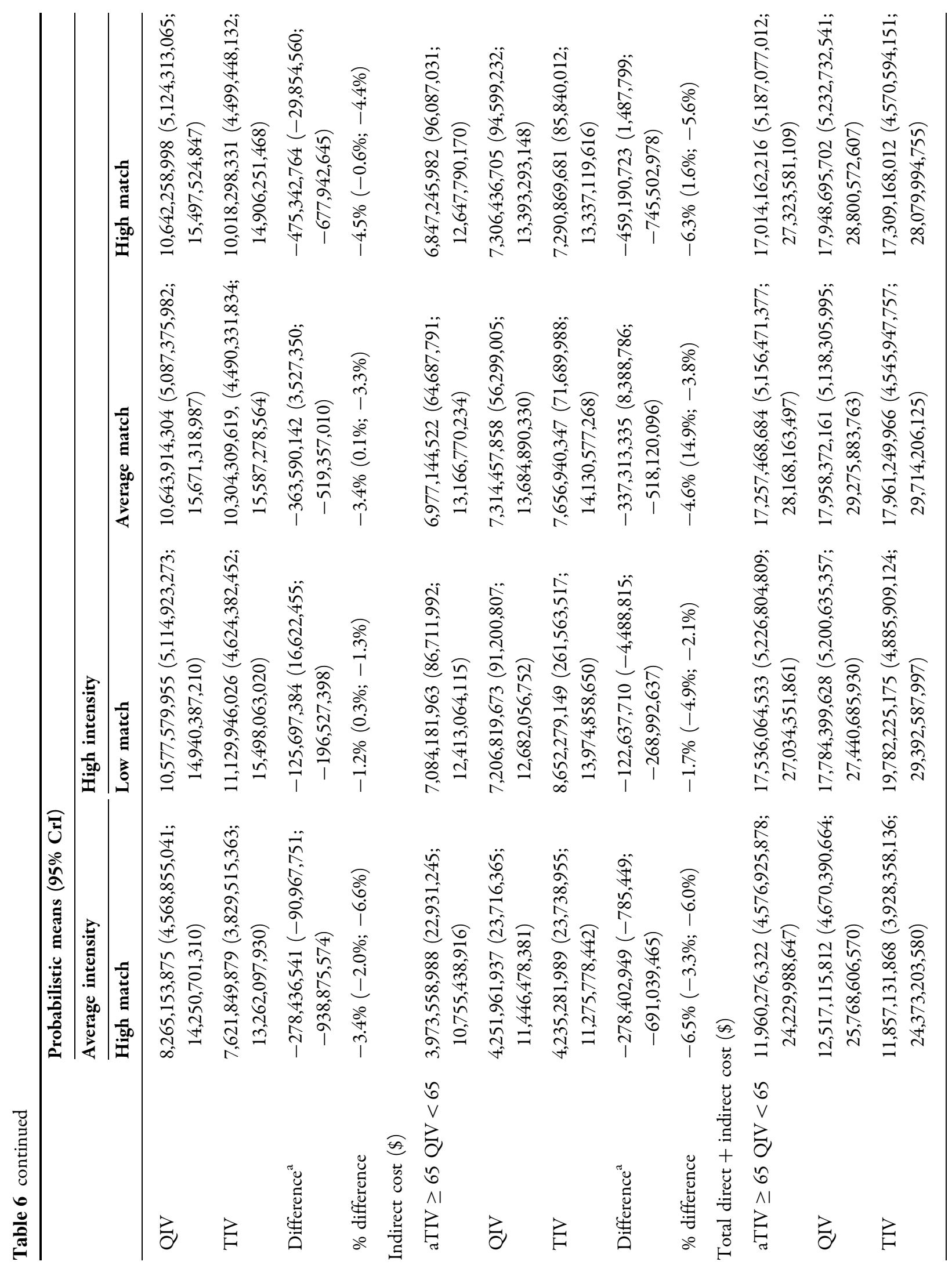




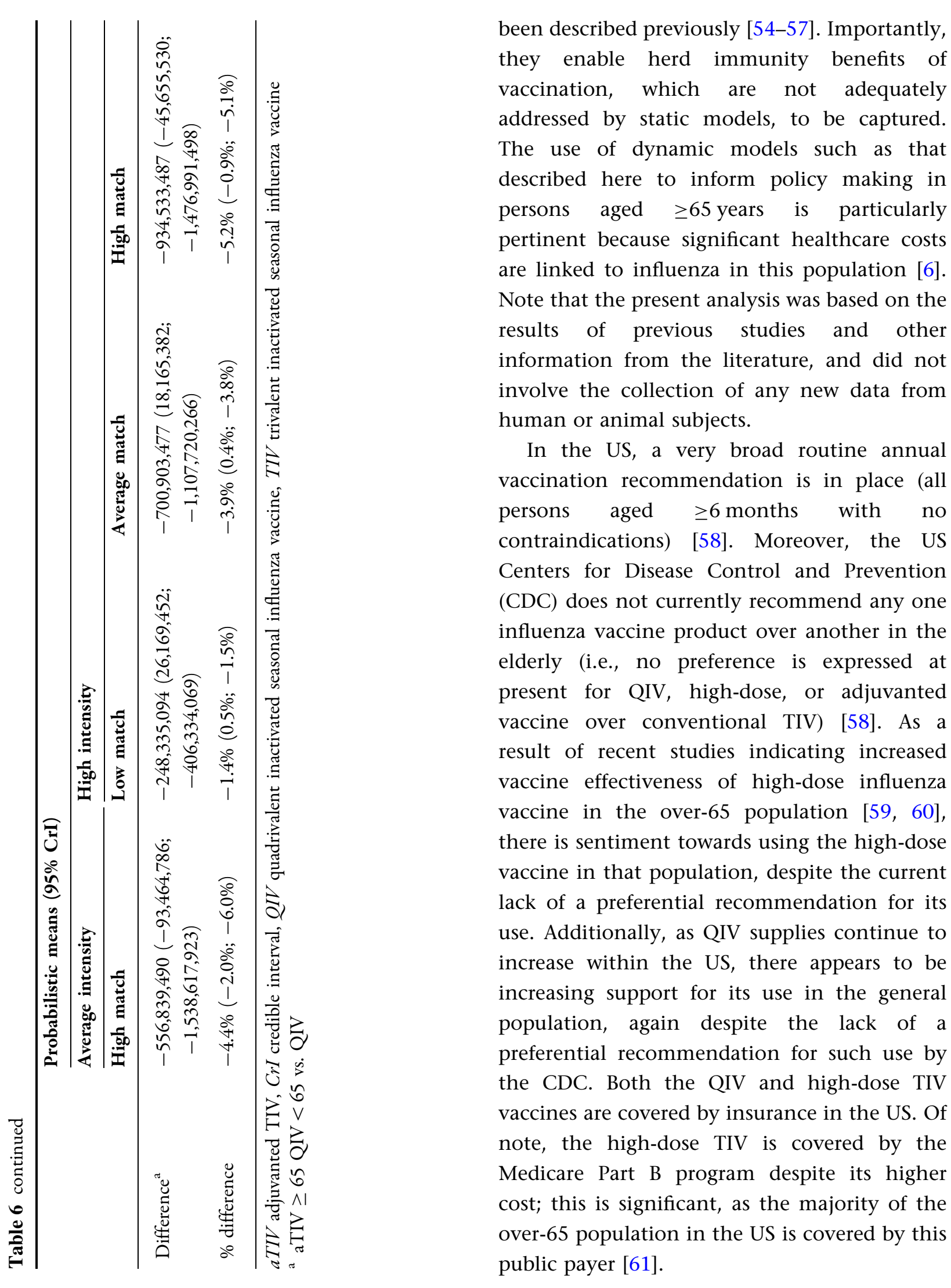


The effectiveness of current vaccination recommendations in the elderly is under debate, as conventional vaccines are reported to provide suboptimal protection in this group [7]. High-dose TIV uptake in the elderly is expected to increase in the US and other countries in the near future, but there remains a need for additional vaccines offering enhanced immunogenicity in this population [62].

Adjuvantation of inactivated vaccines (e.g., with oil-in water adjuvants) has the potential to address unmet influenza vaccination needs in the elderly $[16,62]$, and has been reported to provide better cross-reactivity against mismatched strains than conventional vaccines in the elderly in several studies [26, 63-65]. The potential for improved outcomes with adjuvanted vaccine was apparent from the model, which showed probabilistic mean reductions in clinical outputs and overall costs relative to QIV even in modeled low match seasons. The breakeven analysis carried out after the main simulations suggested that QIV would only offer incremental benefit over aTIV in the elderly if the proportion of mismatched strains in circulation was as high as $54.5 \%$ or above, a level which has not been observed in the US over the last 15 years.

Of note, high-dose TIV has been associated with a $24.2 \%$ increase in efficacy against laboratory-confirmed influenza [59] and a $22 \%$ increase in effectiveness for preventing probable influenza infections and hospitalizations in persons aged $\geq 65$ years [60]. This suggests that economic comparisons of high-dose TIV with other inactivated influenza vaccine options using the model would be of considerable interest and could be the subject of a future analysis. For now, and on the basis of the data and assumptions built into the present model and the above observations with high-dose
Fig. 4 Graphic summary of the main clinical and life-year outputs of the model. All values are probabilistic means (see Tables 4 and 5 for 95\% credible intervals for probabilistically modeled values). aTIV adjuvanted TIV, $Q A L Y$ quality-adjusted life-year, $Q I V$ quadrivalent inactivated seasonal influenza vaccine, $T I V$ trivalent inactivated seasonal influenza vaccine

vaccine, we would expect such an analysis to yield results comparable to those obtained here with aTIV in subjects aged $\geq 65$ years. Such a hypothesis would require future confirmation, however.

As reported above, the potential impact of aTIV versus QIV on costs was split evenly between direct and indirect savings. Direct cost savings were derived from reduced hospitalizations among the elderly, who experience increased serious influenza complication rates over younger populations [27]. Indirect cost savings accrued mainly from the impact on younger working relatives/ associates, who are likely to experience work interruptions due to the need for visitation and care giving, with accompanying losses of productivity [27]. Sensitivity analysis of vaccine prices in the average intensity, average match scenario indicated that aTIV could be marginally cost saving relative to QIV at current Medicare prices.

The importance of indirect protection of older persons through herd immunity effects is illustrated by experience of influenza outbreaks in residential institutions [5]. As mentioned earlier, the structure of the SIR model on which the analysis was based accounts for herd immunity effects, through which the elderly benefit from decreased transmission of influenza among the broader population as a result of vaccination [16].

The data used to populate the model were subject to a number of limitations. Mannino et al.'s study [25] demonstrating the 25\% 


\section{(a) Cases}

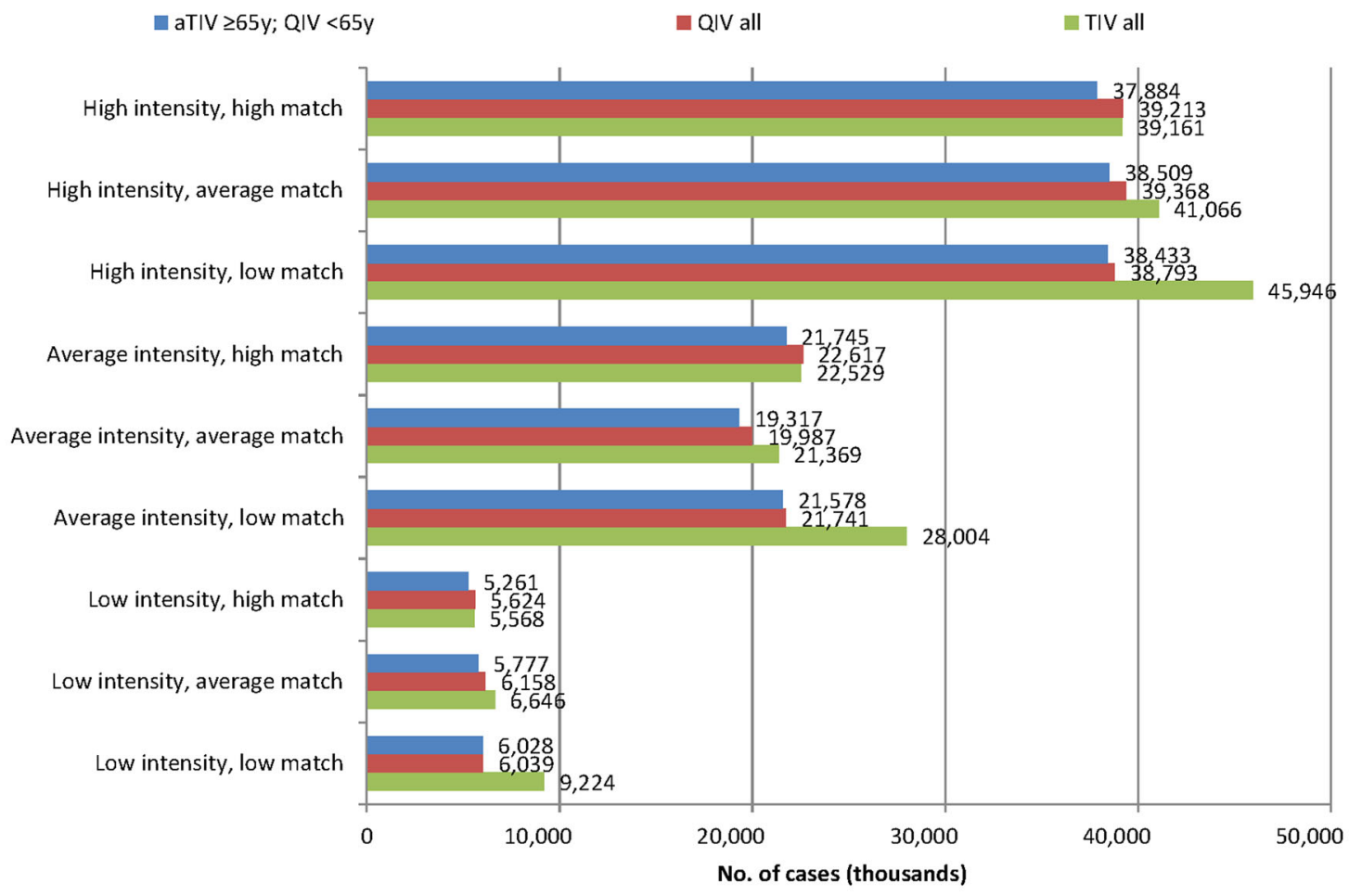

\section{(b) Hospitalizations}

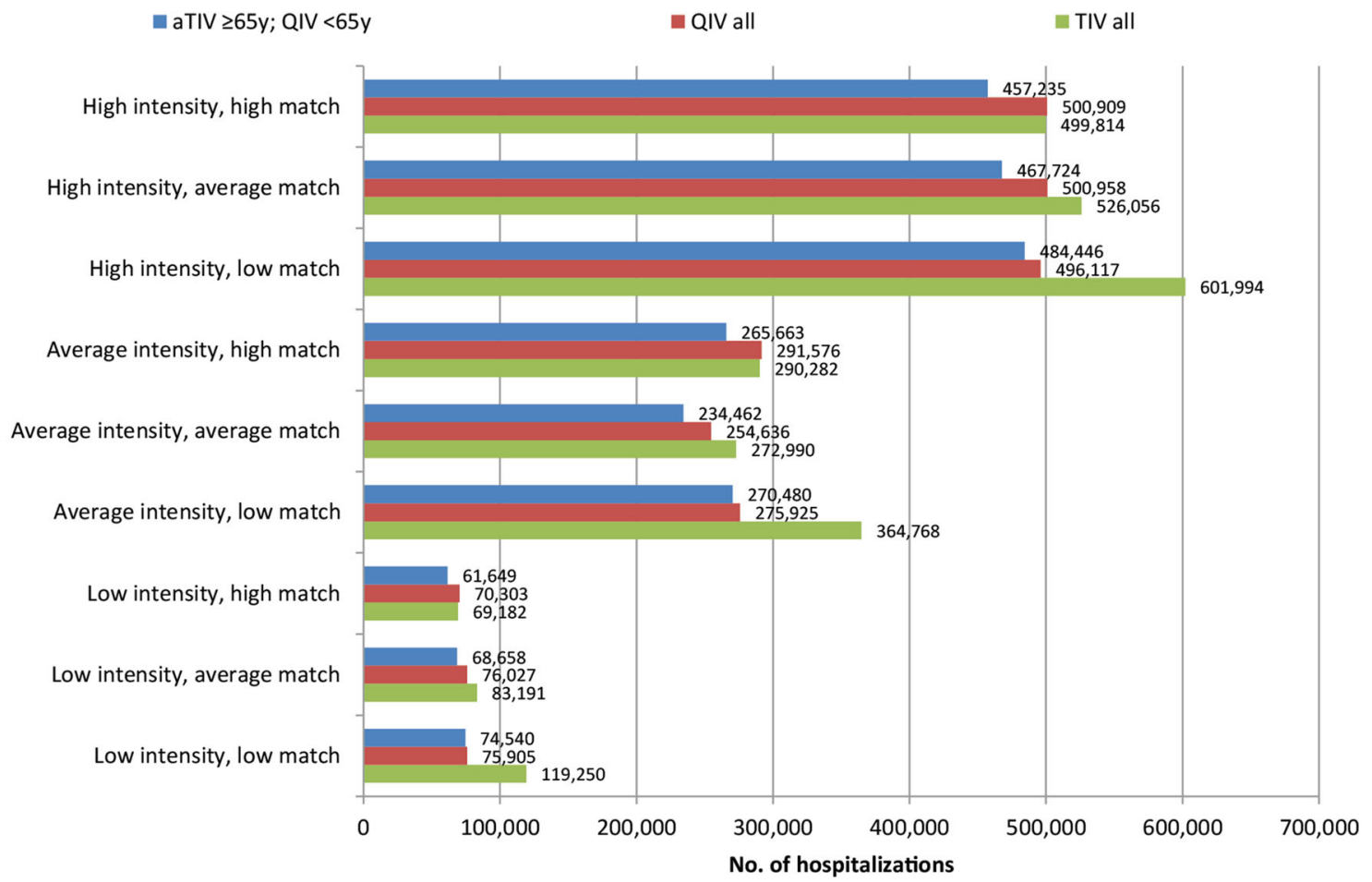




\section{(c) Deaths}

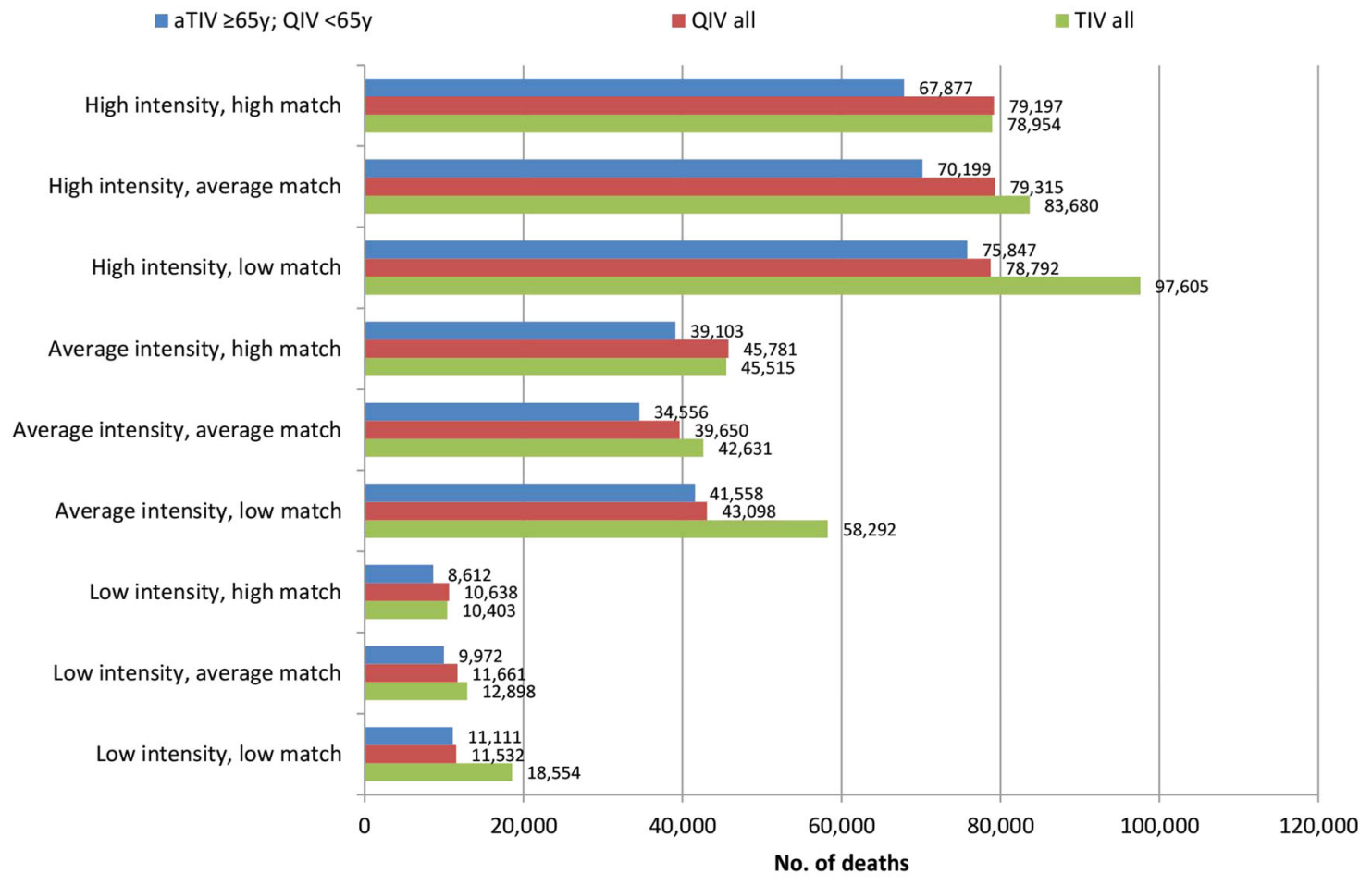

\section{(d) Life years lost}

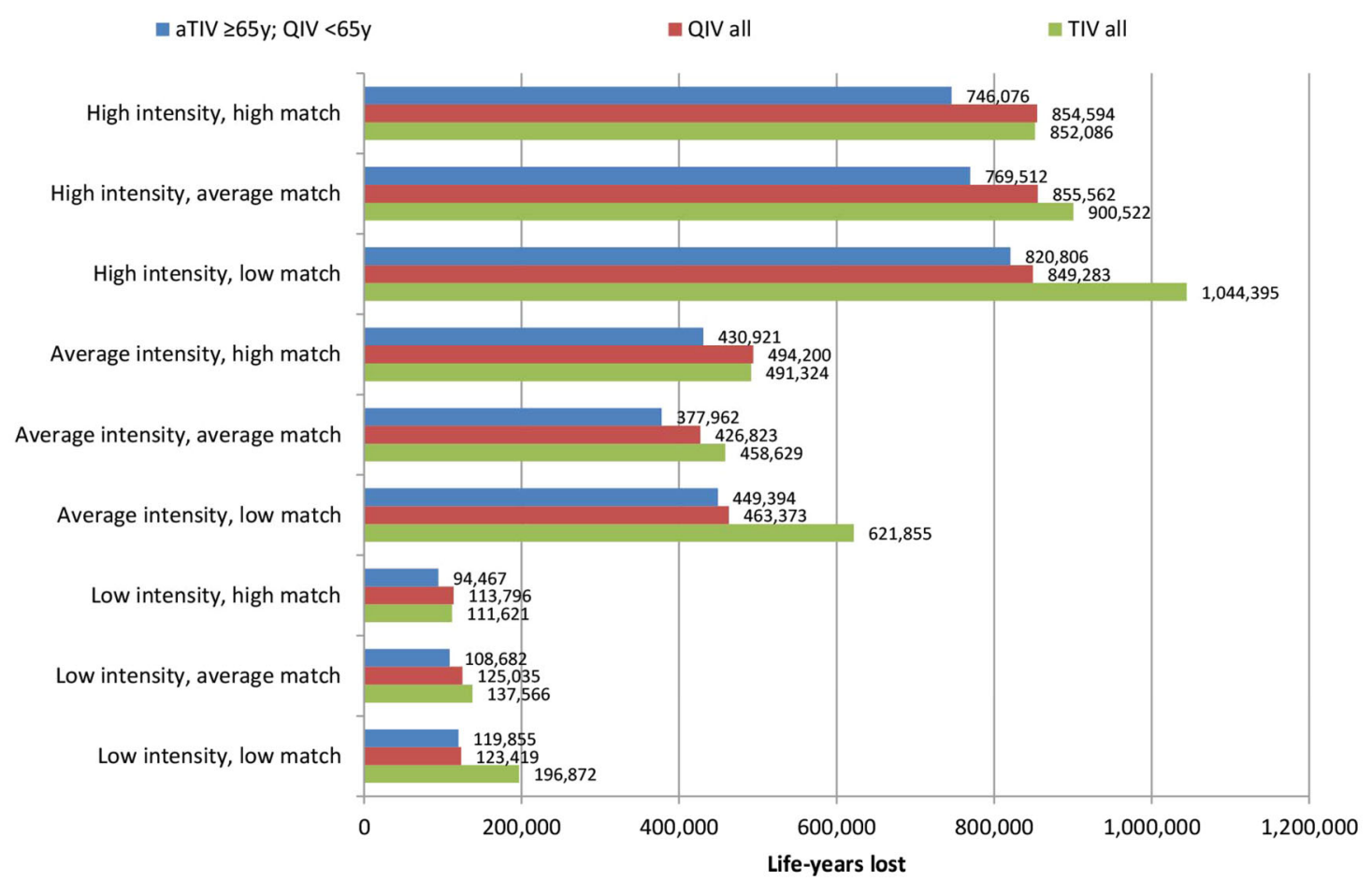

Fig. 4 continued 
(e) QALYs lost

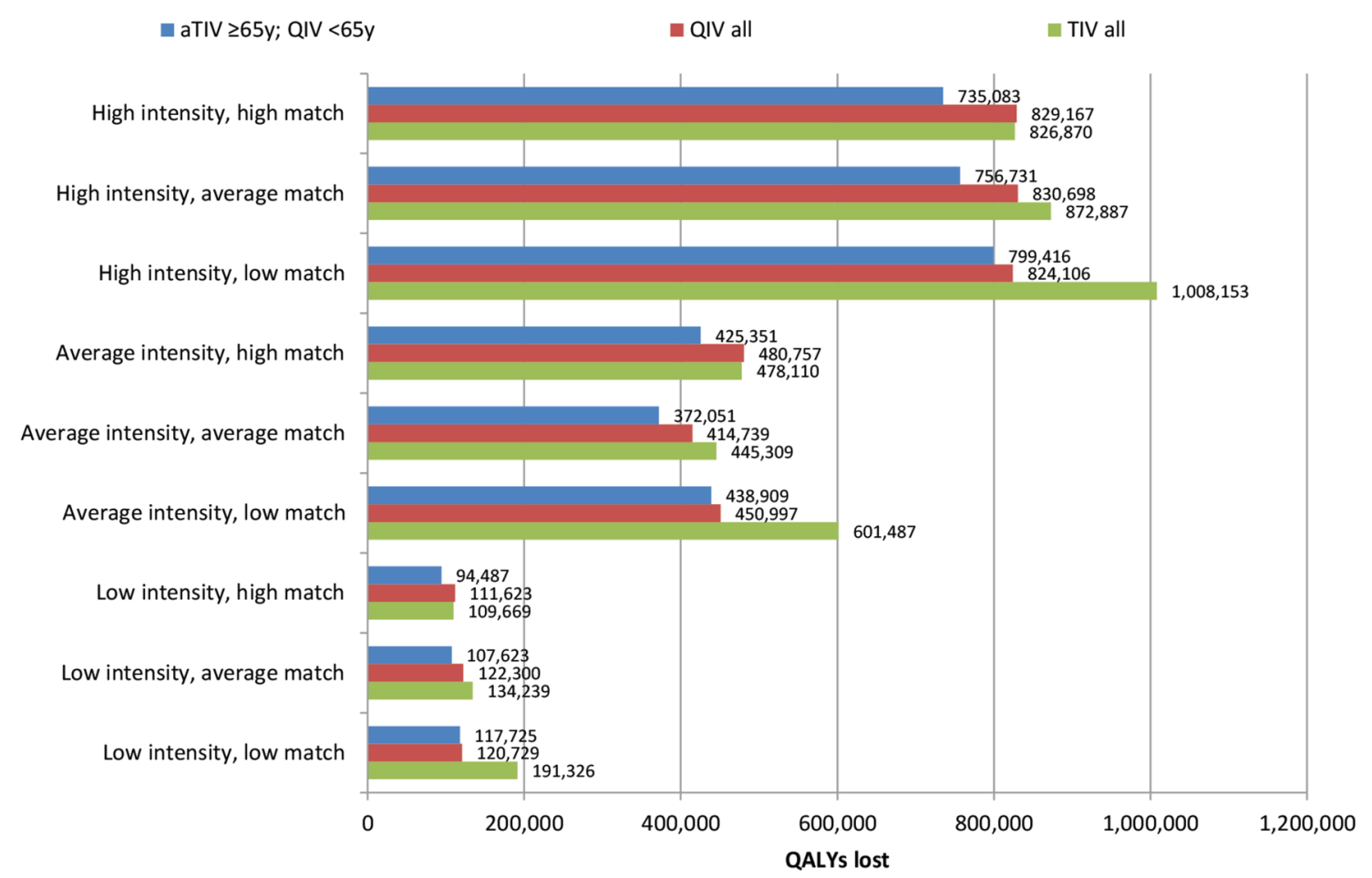

Fig. 4 continued

reduction in risk of hospitalization for influenza or pneumonia versus TIV in adults aged $\geq 65$ years was observational only, did not assess aTIV's impact on the reduction of influenza cases, and did not compare aTIV directly with QIV [25]. There was no stratification other than age in the model, with no account taken of chronic disease status or residential/contact status. The model does not account for adverse effects of influenza vaccination. In addition, costs were treated deterministically in the model even though significant variation occurs in clinical practice. Despite these limitations, the findings are in line with what would be expected for a vaccine offering enhanced immunogenicity that has a potentially positive effect on clinical outcomes and costs relative to conventional vaccines, given the known economic burden of influenza [66] and the public health and economic benefits of vaccination $[66,67]$.

\section{CONCLUSION}

We have developed a dynamic model to assist vaccination policy decisions directing choices between different formulations of seasonal influenza vaccine. The US scenario modeled suggests that vaccination of persons aged $\geq 65$ years with aTIV has the potential to provide clinical and economic benefit relative to QIV and TIV. We recommend further investigation of the clinical and economic impact of aTIV relative to other vaccine formulations in the elderly. 
(a) Total direct cost

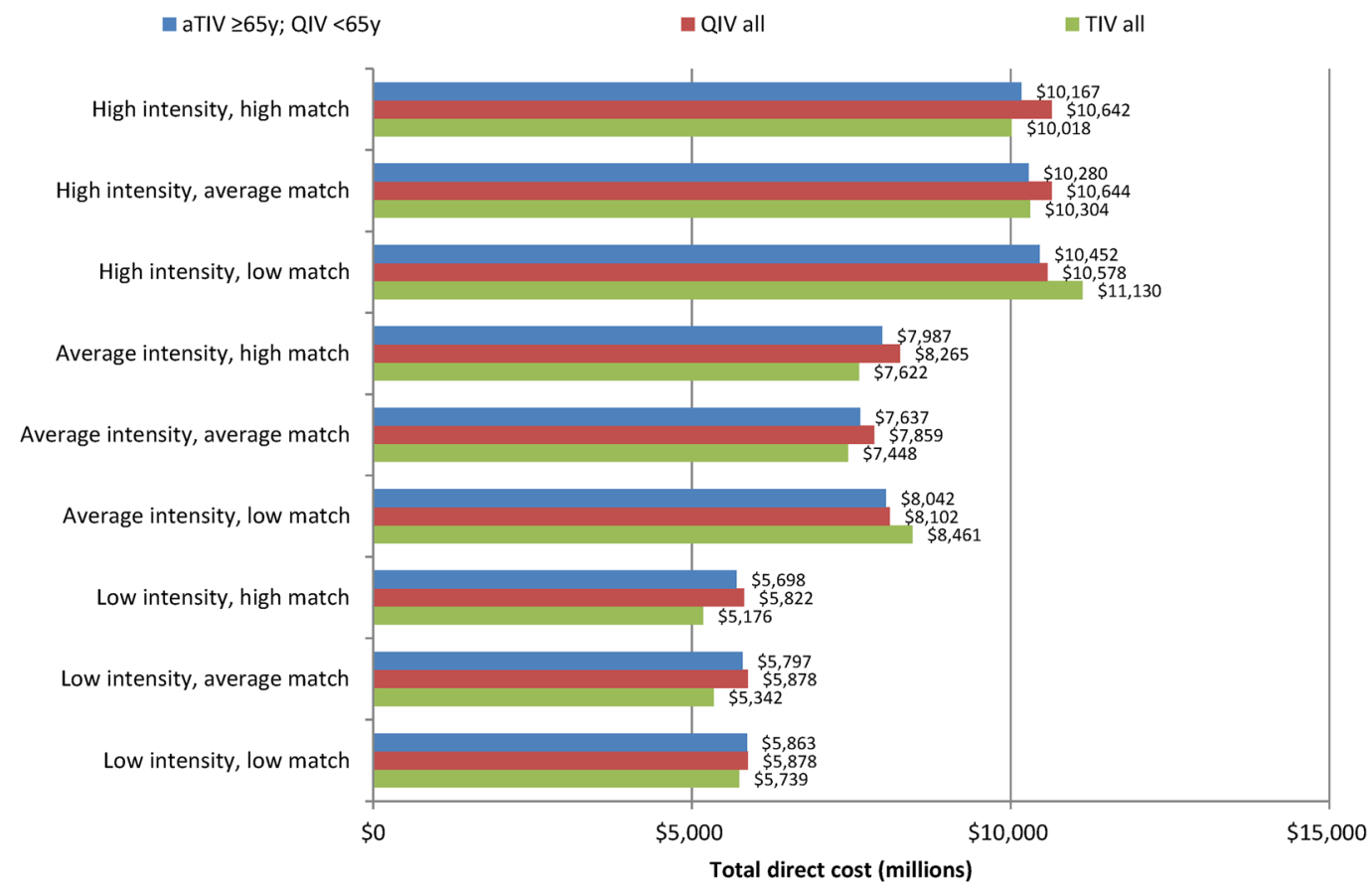

(b) Indirect cost

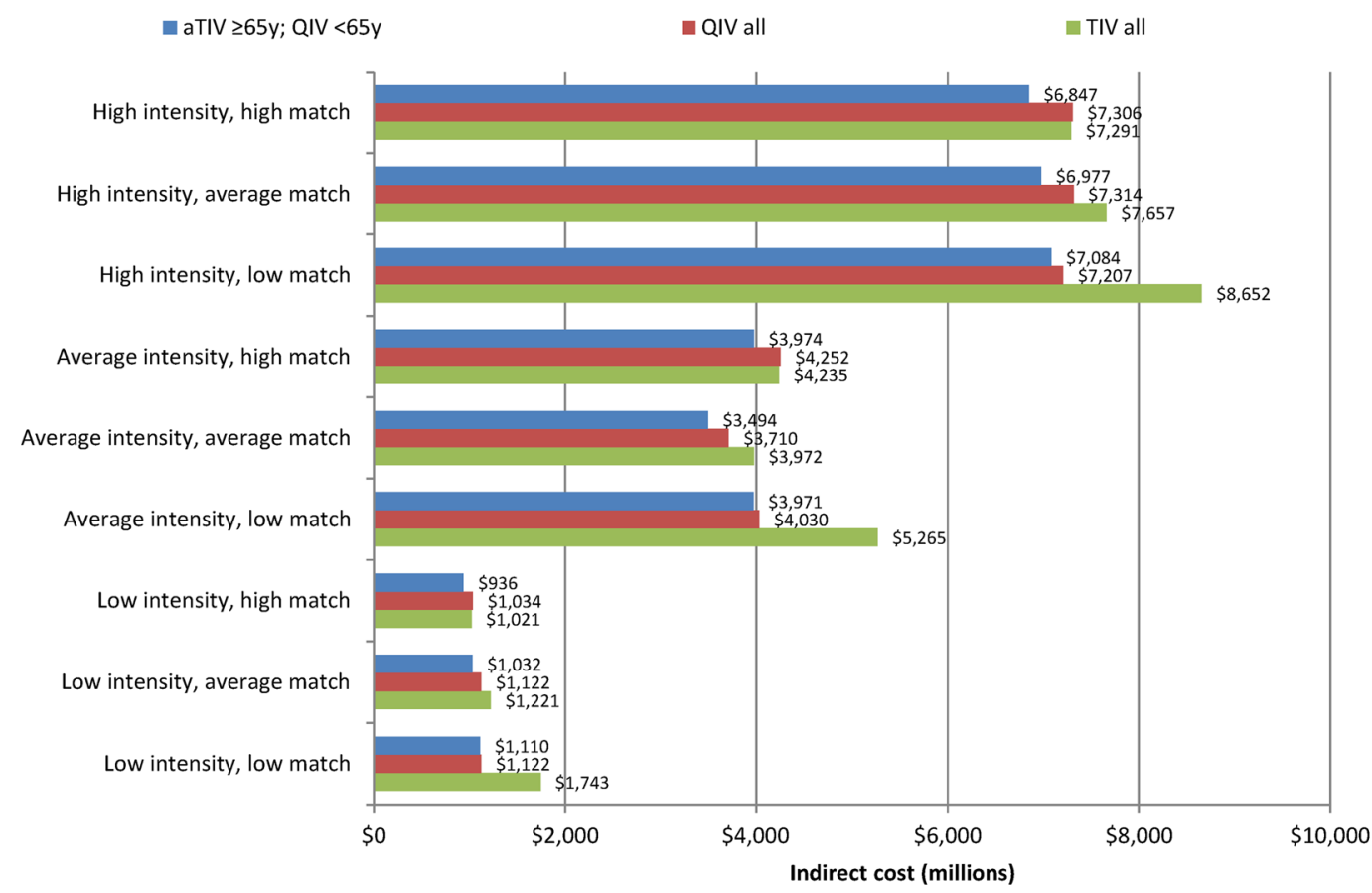

Fig. 5 Graphic summary of the main cost outputs of the model. All values are probabilistic means (see Table 6 for 95\% credible intervals for probabilistically modeled values).
aTIV adjuvanted TIV, QIV quadrivalent inactivated seasonal influenza vaccine, $T I V$ trivalent inactivated seasonal influenza vaccine 
(c) Total and indirect cost

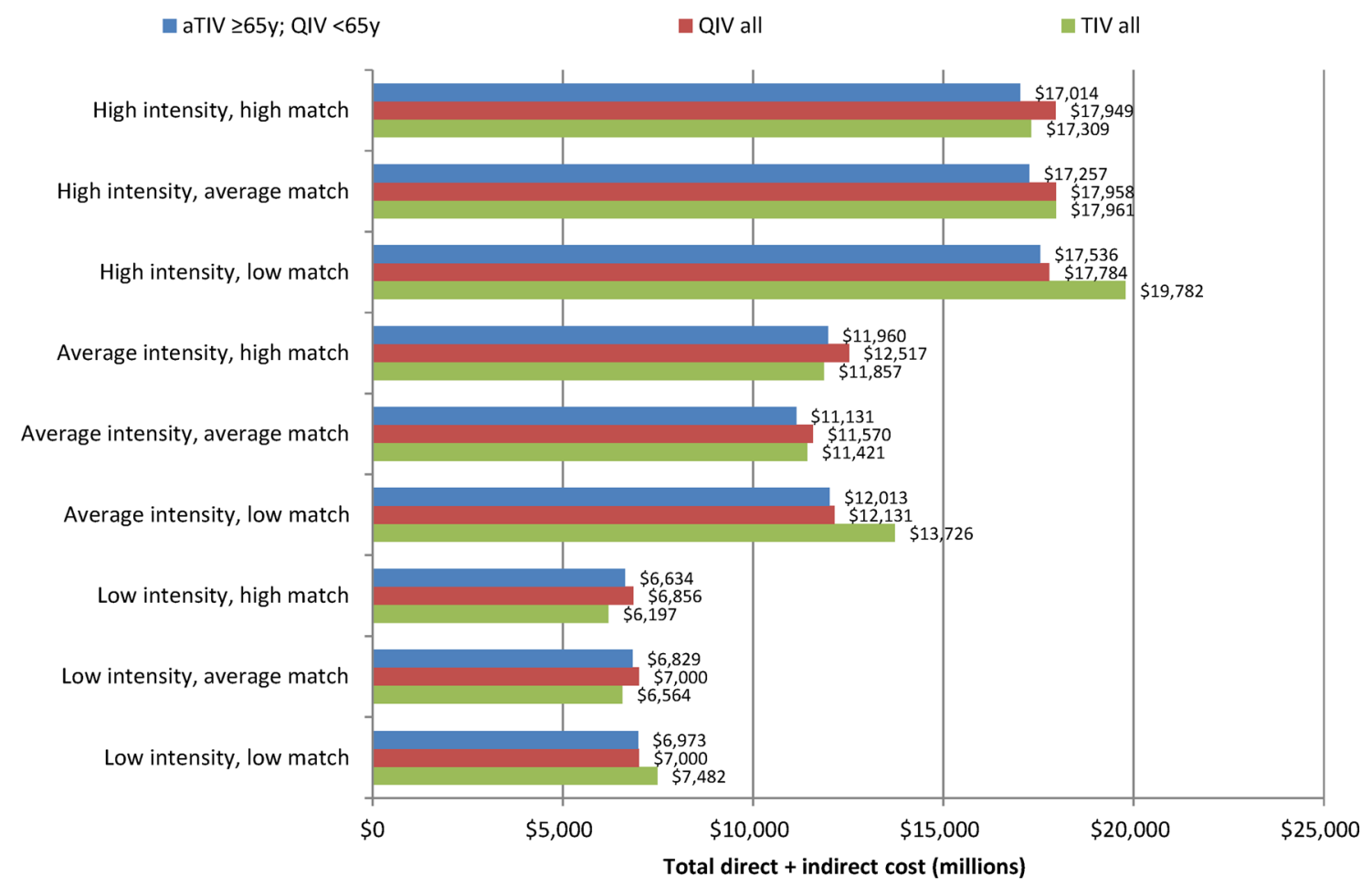

Fig. 5 continued

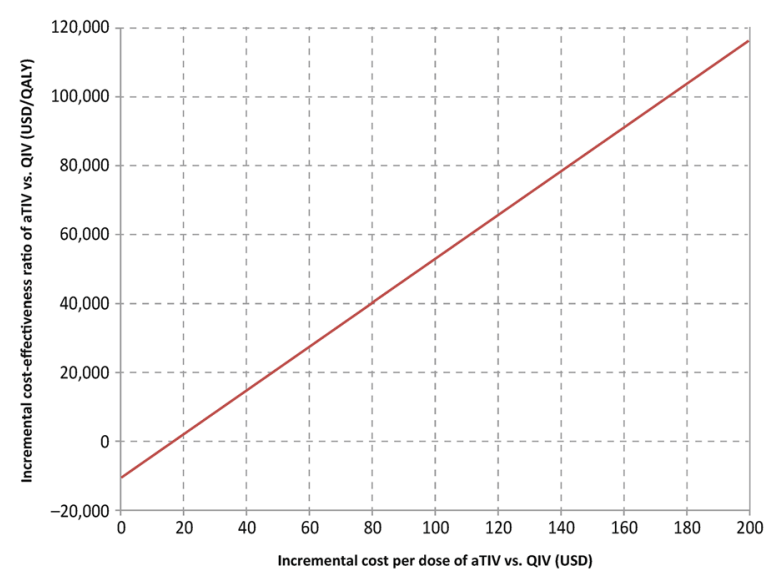

Fig. 6 Univariate sensitivity analysis of relative vaccine prices in the average intensity, average match scenario. $a T I V$ adjuvanted trivalent inactivated influenza vaccine, QALY quality-adjusted life-year, QIV quadrivalent inactivated influenza vaccine

\section{ACKNOWLEDGMENTS}

All named authors meet the International Committee of Medical Journal Editors (ICMJE) criteria for authorship for this manuscript, take responsibility for the integrity of the work as a whole, and have given final approval to the version to be published. Medical Writing support for the development of this manuscript was provided by Christopher Dunn and Scott Malkin at Gardiner-Caldwell Communications, Macclesfield, UK. Funding for this support was provided by Novartis Vaccines. Modeling support for the analyses described in this manuscript was provided by 
MAPI Values, Boston, MA, USA. Funding for this support was provided by Novartis Vaccines.

Conflict of interest. Mark Mullikin, Norbert Farkas, and Eckhardt Petri declare that they are current or former employees of Novartis Vaccines. Jeroen P. Jansen and Marc Van Ranst have no conflict of interest. Litjen Tan has served on scientific advisory boards for Pfizer, Merck, Novartis, Temptime Corp., and TruMedSystems within the past year. All honoraria for these services were donated directly to the Immunization Action Coalition.

Compliance with ethics guidelines. The analysis in this article is based on previously conducted studies, and does not involve any new studies of human or animal subjects performed by any of the authors.

Open Access. This article is distributed under the terms of the Creative Commons Attribution-NonCommercial 4.0 International License (http://creativecommons.org/licenses/ by-nc/4.0/), which permits any noncommercial use, distribution, and reproduction in any medium, provided you give appropriate credit to the original author(s) and the source, provide a link to the Creative Commons license, and indicate if changes were made.

\section{REFERENCES}

1. Meier CR, Napalkov PN, Wegmüller Y, Jefferson T, Jick H. Population-based study on incidence, risk factors, clinical complications and drug utilisation associated with influenza in the United Kingdom. Eur J Clin Microbiol Infect Dis. 2000;19:834-42.

2. Meyers DG. Myocardial infarction, stroke, and sudden cardiac death may be prevented by influenza vaccination. Curr Atheroscler Rep. 2003;2003(5):146-9.
3. Nicholson KG, Kent J, Hammersley V, Cancio E. Acute viral infections of upper respiratory tract in elderly people living in the community: comparative, prospective, population based study of disease burden. BMJ. 1997;315:1060-4.

4. Thompson WW, Shay DK, Weintraub E, et al. Influenza-associated hospitalizations in the United States. J Am Med Assoc. 2004;292:1333-40.

5. Menec VH, MacWilliam L, Aoki FY. Hospitalizations and deaths due to respiratory illnesses during influenza seasons: a comparison of community residents, senior housing residents, and nursing home residents. J Gerontol A Biol Sci Med Sci. 2002;57:M629-35.

6. Menec VH, Black C, MacWilliam L, Aoki FY. The impact of influenza-associated respiratory illnesses on hospitalizations, physician visits, emergency room visits, and mortality. Can J Public Health Rev Can Santé Publique. 2003;94:59-63.

7. Simonsen L, Fukuda K, Schonberger LB, Cox NJ. The impact of influenza epidemics on hospitalizations. J Infect Dis. 2000;181:831-7.

8. Mullooly JP, Bridges CB, Thompson WW, on behalf of the Vaccine Safety Datalink Adult Working Group, et al. Influenza- and RSV-associated hospitalizations among adults. Vaccine. 2007;25:846-55.

9. Thompson WW, Shay DK, Weintraub E, et al. Mortality associated with influenza and respiratory syncytial virus in the United States. J Am Med Assoc. 2003;289:179-86.

10. Zamparo E, Little D. Immunogenicity and effectiveness of virosomal adjuvanted vaccines against influenza: a brief review of their utility in the elderly population. J Prev Med Hyg. 2011;52:116-9.

11. Viboud C, Miller M. Health benefits of universal influenza vaccination strategy. PLoS Med. 2008;5:e216.

12. McElhaney JE, Zhou X, Talbot HK, et al. The unmet need in the elderly: how immunosenescence, CMV infection, co-morbidities and frailty are a challenge for the development of more effective influenza vaccines. Vaccine. 2012;30:2060-7.

13. Monto AS, Ansaldi F, Aspinall R, et al. Influenza control in the 21st century: optimizing protection of older adults. Vaccine. 2009;27:5043-53.

14. Nichol KL. Efficacy and effectiveness of influenza vaccination. Vaccine. 2008;26(Suppl. 4):D17-22. 
15. Nichol KL. The efficacy, effectiveness and cost-effectiveness of inactivated influenza virus vaccines. Vaccine. 2003;21:1769-75.

16. Simonsen L, Taylor RJ, Viboud C, Miller MA, Jackson LA. Mortality benefits of influenza vaccination in elderly people: an ongoing controversy. Lancet Infect Dis. 2007;7:658-66.

17. Sasaki S, Sullivan M, Narvaez CF, et al. Limited efficacy of inactivated influenza vaccine in elderly individuals is associated with decreased production of vaccine-specific antibodies. J Clin Invest. 2011;121:3109-19.

18. Falsey AR, Treanor JJ, Tornieporth N, Capellan J, Gorse GJ. Randomized, double-blind controlled phase 3 trial comparing the immunogenicity of high-dose and standard-dose influenza vaccine in adults 65 years of age and older. J Infect Dis. 2009;200:172-80.

19. Coudeville L, Andre P, Bailleux F, Weber F, Plotkin S. A new approach to estimate vaccine efficacy based on immunogenicity data applied to influenza vaccines administered by the intradermal or intramuscular routes. Hum Vaccin. 2010;6:841-8.

20. Tricco AC, Chit A, Soobiah C, et al. Comparing influenza vaccine efficacy against mismatched and matched strains: a systematic review and meta-analysis. BMC Med. 2013;11:153.

21. DiazGranados CA, Denis M, Plotkin S. Seasonal influenza vaccine efficacy and its determinants in children and non-elderly adults: a systematic review with meta-analyses of controlled trials. Vaccine. 2012;31:49-57.

22. McKeage K. Inactivated quadrivalent split-virus seasonal influenza vaccine (Fluarix ${ }^{\circledR}$ quadrivalent): a review of its use in the prevention of disease caused by influenza A and B. Drugs. 2013;73:1587-94.

23. De Donato S, Granoff D, Minutello M, et al. Safety and immunogenicity of MF59-adjuvanted influenza vaccine in the elderly. Vaccine. 1999;17:3094-101.

24. Squarcione S, Sgricia S, Biasio LR, Perinetti E. Comparison of the reactogenicity and immunogenicity of a split and a subunit-adjuvanted influenza vaccine in elderly subjects. Vaccine. 2003;21:1268-74.

25. Mannino S, Villa M, Apolone G, et al. Effectiveness of adjuvanted influenza vaccination in elderly subjects in northern Italy. Am J Epidemiol. 2012;176:527-33.

26. Durando P, Icardi G, Ansaldi F. MF59-adjuvanted vaccine: a safe and useful tool to enhance and broaden protection against seasonal influenza viruses in subjects at risk. Expert Opin Biol Ther. 2010;10:639-51.

27. Molinari N-AM, Ortega-Sanchez IR, Messonnier ML, et al. The annual impact of seasonal influenza in the US: measuring disease burden and costs. Vaccine. 2007;25:5086-96.

28. Annemans L. Methods for economic evaluations. In: Health economics for non-economists. Gent: Academia Press; 2008. p. 29-79.

29. Reed C, Meltzer MI, Finelli L, Fiore A. Public health impact of including two lineages of influenza $\mathrm{B}$ in a quadrivalent seasonal influenza vaccine. Vaccine. 2012;30:1993-8.

30. Centers for Disease Control and Prevention (CDC). Update: influenza activity-United States, 2010-11 season, and composition of the 2011-12 influenza vaccine. MMWR Morb Mortal Wkly Rep. 2011;60:705-12.

31. Centers for Disease Control and Prevention (CDC). Update: influenza activity-United States, 2011-12 season and composition of the 2012-13 influenza vaccine. MMWR Morb Mortal Wkly Rep. 2012;61:414-20.

32. Centers for Disease Control and Prevention (CDC). Influenza activity-United States, 2012-13 season and composition of the 2013-14 influenza vaccine. MMWR Morb Mortal Wkly Rep. 2013;62:473-9.

33. Epperson S, Blanton L, Kniss K, on behalf of the Influenza Division NCfI, Respiratory Diseases CDC, et al. Influenza activity-United States, 2013-14 season and composition of the 2014-15 influenza vaccines. MMWR Morb Mortal Wkly Rep. 2014;63:483-90.

34. US Department of Commerce. Current Population Survey (CPS) - a joint effort between the Bureau of Labor Statistics and the Census Bureau-People and Households-US Census Bureau [online document]. US Census Bureau. 2012. http://www. census.gov/cps/. Accessed 27 Oct 2014.

35. Del Valle SY, Hyman JM, Hethcote HW, Eubank SG. Mixing patterns between age groups in social networks. Soc Netw. 2007;29:539-54.

36. Biggerstaff M, Cauchemez S, Reed C, Gambhir M, Finelli L. Estimates of the reproduction number for seasonal, pandemic, and zoonotic influenza: a systematic review of the literature. BMC Infect Dis. $2014 ; 14: 480$.

37. Cowling BJ, Fang VJ, Riley S, Peiris JSM, Leung GM. Estimation of the serial interval of influenza. Epidemiology. 2009;20:344-7. 
38. Fraser C, Donnelly CA, Cauchemez S, on behalf of the WHO Rapid Pandemic Assessment Collaboration, et al. Pandemic potential of a strain of influenza A (H1N1): early findings. Science. 2009;324:1557-61.

39. Centers for Disease Control and Prevention (CDC). Flu vaccination coverage, United States, 2013-14 Influenza Season/FluVaxView/Seasonal Influenza (Flu)/CDC [online document]. 2014. http://www. cdc.gov/flu/fluvaxview/coverage-1314estimates. htm. Accessed 27 Oct 2014).

40. Blyth CC, Jacoby P, Effler PV, on behalf of the WAIVE Study Team, et al. Effectiveness of trivalent flu vaccine in healthy young children. Pediatrics. 2014;133:1218-25.

41. Prosser LA. Cost-effectiveness of Influenza Vaccine [online document]. NIVS, May 16-17, 2012, Atlanta, GA, USA. National Adult Influenza Immun. Summit. http://www.izsummitpartners.org/summit/archive/ 2012-nivs/. Accessed 28 Oct 2014.

42. Talbird SE, Brogan AJ, Winiarski AP, Sander B. Cost-effectiveness of treating influenza-like illness with oseltamivir in the United States. Am J Health Syst Pharm. 2009;66:469-80.

43. Lloyd-Jones D, Adams RJ, Brown TM, on behalf of the American Heart Association Statistics Committee and Stroke Statistics Subcommittee, et al. Heart disease and stroke statistics-2010 update: a report from the American Heart Association. Circulation. 2010;121:e46-215.

44. Lee GM, Salomon JA, LeBaron CW, Lieu TA. Health-state valuations for pertussis: methods for valuing short-term health states. Health Qual Life Outcomes. 2005;3:17.

45. Dyer MTD, Goldsmith KA, Sharples LS, Buxton MJ. A review of health utilities using the EQ-5D in studies of cardiovascular disease. Health Qual Life Outcomes. 2010;8:13.

46. Prosser LA, Ray GT, O’Brien M, Kleinman K, Santoli J, Lieu TA. Preferences and willingness to pay for health states prevented by pneumococcal conjugate vaccine. Pediatrics. 2004;113:283-90.

47. Fryback DG, Dunham NC, Palta M, et al. US norms for six generic health-related quality-of-life indexes from the National Health Measurement study. Med Care. 2007;45:1162-70.

48. ftp.cdc.gov-/pub/health_Statistics/NCHS/ Publications/NVSR/56_09/ [online document] [no date]. http://ftp.cdc.gov/pub/health_Statistics/ NCHS/Publications/NVSR/56_09/. Accessed 15 June 2015.
49. Fisman DN, Tuite AR. Estimation of the health impact and cost-effectiveness of influenza vaccination with enhanced effectiveness in Canada. PLoS One. 2011;6:e27420.

50. Seasonal Flu [online document]. 2015. Flu.gov. http://www.flu.gov/about_the_flu/seasonal/index. html. Accessed 12 June 2015.

51. Centers for Medicare and Medicaid Services. Physician fee schedule. 2014. https://www.cms. gov/Medicare/Medicare-Fee-for-Service-Payment/ PhysicianFeeSched/index.html?redirect $=$ / PhysicianFeeSched/. Accessed 22 July 2015.

52. Centers for Medicare and Medicaid Services. Clinical laboratory fee schedule. 2014. http:// www.cms.gov/Medicare/Medicare-Fee-for-ServicePayment/ClinicalLabFeeSched/clinlab.html. Accessed 22 July 2015.

53. Centers for Medicare and Medicaid Services. Average sales price (ASP) drug pricing files. 2014. http://www.cms.gov/Medicare/Medicare-Fee-forService-Part-B-Drugs/McrPartBDrugAvgSalesPrice/ 2014ASPFiles.html. Accessed 22 July 2015.

54. Nichol KL, Tummers K, Hoyer-Leitzel A, Marsh J, Moynihan M, McKelvey S. Modeling seasonal influenza outbreak in a closed college campus: impact of pre-season vaccination, in-season vaccination and holidays/breaks. PLoS One. 2010;5:e9548.

55. Coburn BJ, Wagner BG, Blower S. Modeling influenza epidemics and pandemics: insights into the future of swine flu (H1N1). BMC Med. 2009; 7:30.

56. Knipl DH, Röst G. Modelling the strategies for age specific vaccination scheduling during influenza pandemic outbreaks. Math Biosci Eng. 2011;8:123-39.

57. Damm O, Eichner M, Rose MA, et al. Public health impact and cost-effectiveness of intranasal live attenuated influenza vaccination of children in Germany. Eur J Health Econ. 2015;16:471-88.

58. Centers for Disease Control and Prevention (CDC). Prevention and Control of Seasonal Influenza with Vaccines/Health Professionals/Seasonal Influenza (Flu) [online document]. 2014. http://www.cdc. gov/flu/professionals/acip/index.htm. Accessed 30 Oct 2014).

59. DiazGranados CA, Dunning AJ, Kimmel M, et al. Efficacy of high-dose versus standard-dose influenza vaccine in older adults. $\mathrm{N}$ Engl $\mathrm{J}$ Med. 2014;371:635-45. 
60. Izurieta HS, Thadani N, Shay DK, et al. Comparative effectiveness of high-dose versus standard-dose influenza vaccines in US residents aged 65 years and older from 2012 to 2013 using Medicare data: a retrospective cohort analysis. Lancet Infect Dis. 2015; 15:293-300.

61. Centers for Medicare and Medicaid Services. Medicare Enrollment-National Trends 1966-2013. Medicare aged and disabled enrollees by type of coverage. All areas, as of July 1, 1966-2013. Hospital and/or Supplementary Medical Insurance, Medicare Enrollment Reports. Baltimore: Centers for Medicare and Medicaid Services; 2014.

62. Datamonitor Healthcare. Influenza vaccines forecast (No. DMKC0107117). London: Informa UK Ltd; 2014.

63. Baldo V, Baldovin $\mathrm{T}$, Pellegrini $\mathrm{M}$, et al. Immunogenicity of three different influenza vaccines against homologous and heterologous strains in nursing home elderly residents. Clin Dev Immunol. 2010;2010:517198.
64. Ansaldi F, Zancolli M, Durando P, et al. Antibody response against heterogeneous circulating influenza virus strains elicited by MF59- and non-adjuvanted vaccines during seasons with good or partial matching between vaccine strain and clinical isolates. Vaccine. 2010;28:4123-9.

65. Orsi A, Ansaldi F, de Florentiis D, et al. Cross-protection against drifted influenza viruses: options offered by adjuvanted and intradermal vaccines. Hum Vaccines Immunother. 2013;9:582-90.

66. Xue Y, Kristiansen IS, de Blasio BF. Modeling the cost of influenza: the impact of missing costs of unreported complications and sick leave. BMC Public Health. 2010;10:724.

67. Preaud E, Durand L, Macabeo B, on behalf of the Vaccines Europe Influenza Working Group, et al. Annual public health and economic benefits of seasonal influenza vaccination: a European estimate. BMC Public Health. 2014;14:813. 